\title{
MICA CONDENSERS AS STANDARDS OF CAPACITY
}

\author{
By Harvey L. Curtis
}

\section{CONTENTS}

I. INTRODUCTION . . . . . . . . . . . . . . . . . . . . . . 432

II. Methods of Measurement of Capacity . . . . . . . . . . . . 433

I. Absolute measurement of the capacity of a mica condenser..... 433

2. Alternating-current comparison of capacities............. 435

(a) Series-resistance method ................... 435

(b) Series-inductance method . .................. 436

(c) Advantages and disadvantages of each method ........ 438

(d) A direct-reading capacity bridge................. 439

(e) The choice of resistances................... 439

3. Alternating-current comparison of a capacity and an inductance... 440

4. Direct-current comparison of capacities ................. 44 I

(a) The differential galvanometer method ............ 44 I

(b) The method of mixtures................... 44 I

5. Methods of "stepping up" ..................... 445

6. Measurement of absorption. . . . . . . . . . . . . . . . 446

III. External Conditions AfFecting the Capacity . . . . . . . . . . . . . 447

7. The temperature coefficient . . . . . . . . . . . . . . . . 447

8. The pressure coefficient . . . . . . . . . . . . . . . . . . . . . . $44^{8}$

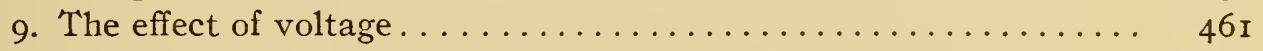

IV. The Stability of Mica Condensers. . . . . . . . . . . . . . . . 463

V. The Relationship Between the Different Apparent Capacities. . 467

IO. Qualitative relations . . . . . . . . . . . . . . . . 468

II. Application of Maxwell's theory of absorption to direct-current measurements...................... 468

I2. Application of Maxwell's theory to alternating-current measure-

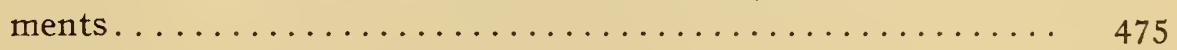

I3. Determination of the geometric capacity ............. 478

14. Effect of leakage.......................... 482

I5. Possible sources of error in the measurement of the geometric

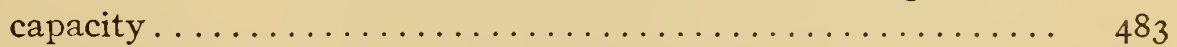

16. Empirical formulas ....................... $44_{4}$

I 7. Cases of practical importance.................... 487

VI. SUMMARY . . . . . . . . . . . . . . . . . . . . . . 487 


\section{INTRODUCTION}

In the choice of a condenser to be used as a standard of capacity, the following things need to be considered: Its stability, the effect of its absorption upon the measured capacity, and the influence of such external conditions as temperature, atmospheric pressure, and the applied voltage upon this capacity. The requirements necessary to satisfy the conditions thus imposed are only approximately fulfilled by air condensers, as has been shown by Giebe, ${ }^{1}$ and it is the purpose of this investigation to show how nearly they are met in the case of mica condensers. Giebe found that with air condensers the effect of absorption and the influence of changes of voltage are not measurable, while the temperature and pressure coefficients may be readily determined. However, he found that, although his condensers were constructed with special reference to the attainment of stability, there sometimes occurred an unaccountable change of one part in ten thousand in the capacity within an interval of two or three days, and it is well known that ordinary air condensers often show much greater changes than this. Moreover, except for the smallest capacities, an air condenser is of necessity very bulky, so that it can be satisfactorily used in comparatively few cases.

A mica condenser is of much more convenient size, but the absorption produces a very marked effect upon the measured capacity. However, it has been shown by Zeleny, ${ }^{2} \mathrm{Hill},{ }^{3}$ and others that this effect is always the same under the same conditions, so that the apparent capacity by any method of measurement is perfectly definite. The effect of temperature upon the capacity has been investigated by Bedell, ${ }^{4}$ Terry, ${ }^{5}$ and others, but the author has not found any investigation of the effect of temperature and pressure upon the stability of mica condensers nor of the relation between the effects of absorption and temperature upon the capacity. This investigation was undertaken to determine the conditions under which a condenser is most stable, to find out as much as possible concerning the effect of absorption on the measured capacity, and to determine the effect of such external condi-

\footnotetext{
${ }^{1}$ Zeit. für Instr., 29, p. 30 I ; 1909.

2 Phys. Rev., 22, p. 65 ; 1906.

${ }^{3}$ Phys. Rev., 26, p. 400; 1908.

${ }^{4}$ Phys. Rev. 1, p. 81; 1893.

${ }^{5}$ Phys. Rev., 21, p. I93; I905.
} 
tions as temperature, atmospheric pressure, and applied voltage upon the different apparant capacities. In this way it was hoped to determine to what extent a mica condenser can be considered a satisfactory standard of capacity.

\section{METHODS OF MEASUREMENT OF CAPACITY}

The methods of measurement of capacity used in this investigation can be classed under four heads: (I) Absolute measurement in terms of resistance and time; (2) alternating current comparison of one capacity with another; (3) alternating current comparison of a capacity with an inductance; (4) direct current comparison of two capacities. Beside these measurements, determinations of the phase difference, of the absorption, and of the insulation resistance have been frequently made. A complete discussion of all these methods is unnecessary, but in the following is given a description of the distinctive features of the methods which have been used in this investigation.

\section{ABSOLUTE MEASUREIMENT OF THE CAPACITY OF A MICA CONDENSER}

The method most often used in the absolute measurement of a capacity is Maxwell's bridge method. ${ }^{6}$ A description of the way in which it is employed in this laboratory has already been published. ${ }^{7}$ In the case of condensers which show no absorption this is capable of a high degree of accuracy; but with condensers in which there is appreciable absorption the apparent capacity will not only depend on the number of charges and discharges per second, but will also depend upon the length of contact of the charge and discharge brushes, even though the number of charges and discharges per second remains constant. The importance of this last statement will be realized when one considers that the absorption current is relatively large during the first hundredth of a second after the charging begins, so that when the total length of charge is approximately threethousandths of a second, a variation in the length of charge of 
a thousandth of a second will produce an appreciable change in the quantity of electricity flowing into the condenser. Tables I and II indicate the magnitude of these effects in the case of good mica condensers.

\section{TABLE I}

To Show the Effect of the Number of Charges and Discharges per Second upon the Apparent Capacity of a Mica Condenser

\begin{tabular}{c|c|c|c}
\hline Condenser & Capacity at 50 cycles & Capacity at 100 cycles & $\begin{array}{c}\text { Change in parts in a hun- } \\
\text { dred thousand }\end{array}$ \\
\hline No. 6 & $0.500052 \mathrm{mf}$ & $0.499772 \mathrm{mf}$ & 56 \\
No. 7 & 0.498666 & 0.498378 & 58 \\
\hline
\end{tabular}

Both of these are condensers of moderate absorption.

\section{TABLE II}

To Show the Effect of Length of Charge and Discharge on the Apparent Capacity as Measured by Maxwell's Bridge

\begin{tabular}{|c|c|c|c|}
\hline Condenser & $\begin{array}{l}\text { Length of contact of charg- } \\
\text { ing brush, seconds }\end{array}$ & $\begin{array}{l}\text { Length of contact of dis- } \\
\text { charging brush, sec- } \\
\text { onds }\end{array}$ & $\begin{array}{l}\text { Relative change in capac- } \\
\text { ity in parts in a hun- } \\
\text { dred thoussand }\end{array}$ \\
\hline No. 6 & 0.0019 & 0.0025 & 0 \\
\hline A medium-grade & 0.0025 & “ & 22 \\
\hline mica & 0.0036 & “ & 36 \\
\hline \multirow{3}{*}{$\begin{array}{c}5471 \mathrm{A0} .1 \\
\text { A high-grade mica }\end{array}$} & 0.0018 & 0.0025 & 0 \\
\hline & 0.0021 & " & 8 \\
\hline & 0.0025 & “ & 11 \\
\hline \multirow[t]{4}{*}{$5471 \mathrm{~A} 0.1$} & 0.0025 & 0.0014 & 11 \\
\hline & “ & 0.0018 & 10 \\
\hline & “ & 0.0021 & 5 \\
\hline & “ & 0.0026 & 0 \\
\hline \multirow[t]{2}{*}{ Air } & 0.0016 & 0.0014 & 0 \\
\hline & 0.0025 & 0.0025 & 0 \\
\hline
\end{tabular}


It follows that the apparent capacity of a mica condenser as determined by Maxwell's method may be made very definite by specifying the number of charges and discharges per second, and the length of time of charge and of discharge. Nevertheless, it is seldom that it will be of use in measurements of precision, as it is not often that the capacity under these conditions is desired. However, as will be shown later, the capacity determined by this method differs but little from the alternating-current capacity at the same frequency.

The method actually used in obtaining the apparent capacity of a mica condenser is to measure the capacity of an air condenser by Maxwell's method and then to compare it immediately with the mica condenser by whatever method is desired. This gives the absolute value of the apparent capacity. When the mica condenser has nearly the same capacity as the air condenser it will give very satisfactory results, but in case the mica condenser has a capacity more than 50 per cent greater than the capacity of the air condenser, it is more satisfactory to compare two or more mica condensers of the same nominal capacity as the air condenser with the air condenser and then combine these to give the desired value. This requires an investigation of the methods of "stepping up," which will be discussed later.

\section{ALTERNATING-CURRENT COMPARISONS OF CAPACITY}

Grover ${ }^{8}$ has given a very complete description of the various bridge methods for comparing two condensers by alternating current. The two most valuable ones are $(a)$ the series resistance method and $(b)$ the series-inductance method.

(a) The Series-Resistance Method

In the series-resistance method the current in the two arms of the bridge is made to agree in phase by means of the resistances $r_{1}$ and $r_{2}$ in series with the condensers. This is necessary since, on account of the absorption, the phase angles of the condensers are not $90^{\circ}$, but they behave as though resistances $\rho_{1}$ and $\rho_{2}$ were in series with perfect condensers of capacities $C_{1}$ and $C_{2}$. The quantities $\rho_{1}$ and $\rho_{2}$ are called fictitious resistances. In order that there may be a balance when a vibration galvanometer or a telephone 


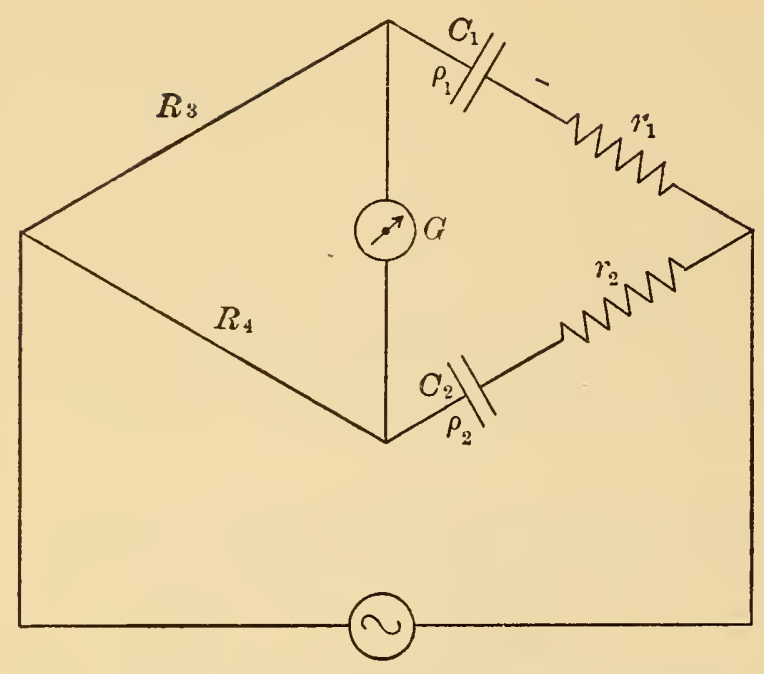

NOMENCLATURE

$r_{1}, r_{2}, R_{3}$, and $R_{4}$ are resistances;

$C_{1}$ and $C_{2}$ are capacities;

$\rho_{1}$ and $\rho_{3}$ are the fictitious resistances of the condensers.

Fig. 1.-Diagram of the series-resistance method of comparing capacities

is used as an indicating instrument, the following relations must hold.

$$
\frac{R_{3}}{R_{4}}=\frac{\rho_{1}+r_{1}}{\rho_{2}+r_{2}}=\frac{C_{2}}{C_{1}}
$$

This shows that there are two independent adjustments which must be made to obtain a balance.

NOMENCLATURE

$R_{3}$ and $R_{4}$ are resistances;

$L_{3}$ and $L_{4}$ are inductances;

$C_{1}$ and $C_{2}$ are capacities;

$\rho_{1}$ and $\rho_{2}$ are the fictitious resist-

ances of the condensers.

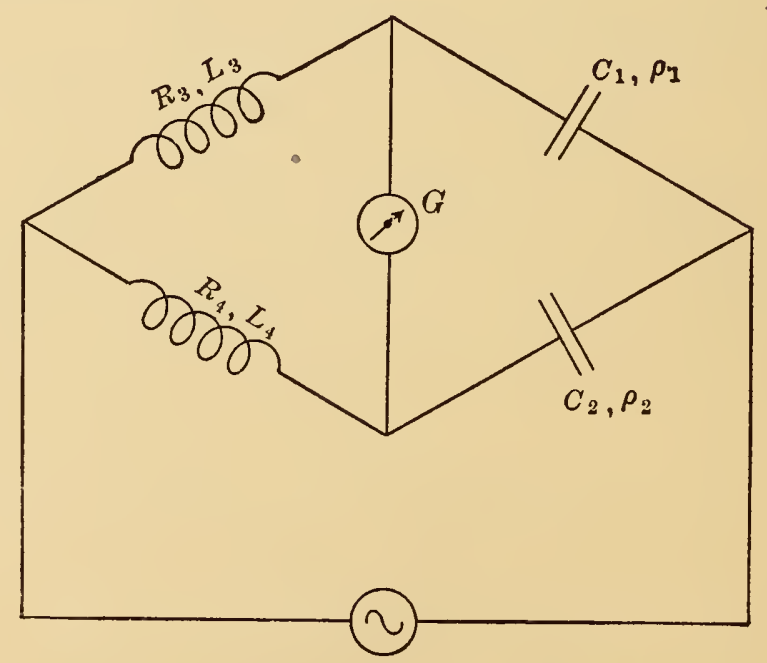

Fig. 2.-Diagram of the series-inductance method of comparing capacities

(b) The Series-Inductance Method

In the series-inductance method the currents are made to agree in phase by means of inductances placed in the resistance 
arms of the bridge. It is necessary that one of these shall be a variable inductance.

A balance is obtained by successive variations of

and

$$
\begin{aligned}
& R_{3} \text { or } R_{4} \text { and } L_{3} \text { or } L_{4} \text {. Then } \\
& \frac{C_{1}}{C_{2}}=\frac{R_{4}}{R_{3}}+\frac{p^{2} C_{1}}{R_{3}}\left(L_{3} \rho_{2}-L_{4} \rho_{1}\right)
\end{aligned}
$$

$$
R_{3} \rho_{2}-R_{4} \rho_{1}=\frac{L_{4}}{C_{1}}-\frac{L_{3}}{C_{2}}
$$

where $p=2 \pi$ times the frequency.

In the case of mica condensers, if $L_{3}$ and $L_{4}$ are kept as small as practicable, the correction term of (2) is negligible ${ }^{9}$ even at high frequencies. Hence

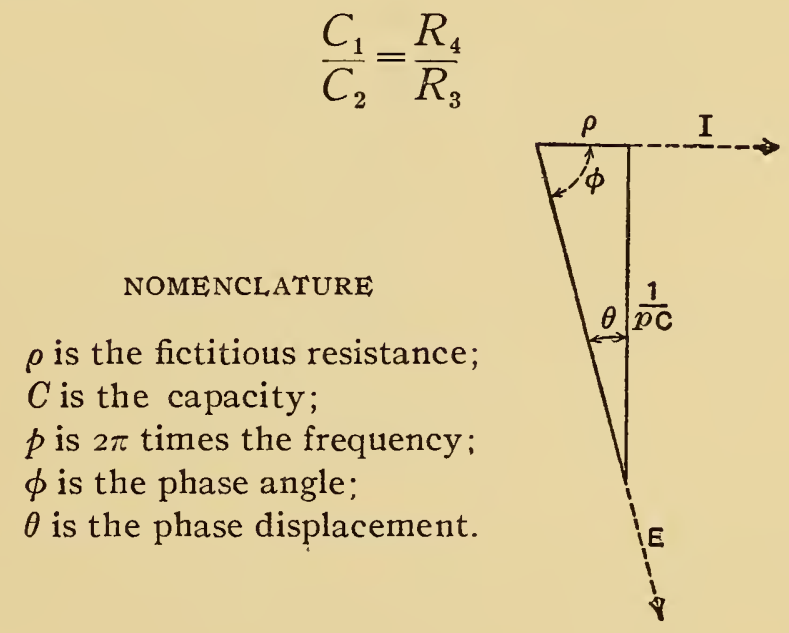

Fig. 3.

The relation between the current and electromotive force may be represented by the usual diagram (see Fig. 3). If $\rho$ and therefore $\theta$ is small, $\tan \theta=\theta=p C \rho$. Hence by multiplying equation (3) by $\frac{p C_{1} C_{2}}{R_{3} R_{4}}$ and noticing that by equation (4) $\frac{C_{1}}{R_{4}}=\frac{C_{2}}{R_{3}}$

$$
\begin{aligned}
p C_{2} \rho_{2}-p C_{1} \rho_{1} & =p\left(\frac{L_{4}}{R_{4}}-\frac{L_{3}}{R_{3}}\right) \\
\theta_{2}-\theta_{1} & =p\left(\frac{L_{4}}{R_{4}}-\frac{L_{3}}{R_{3}}\right)
\end{aligned}
$$


If $C_{1}$ is an air condenser having negligible internal resistance, $\theta_{1}$ is zero, and this equation can be used to determine the phase difference $\theta_{2}$ of a mica condenser with which it is being compared. It has been found more valuable to determine the phase difference than the fictitious resistance of a condenser, since the phase difference varies over a comparatively small range with change of frequency, while the variation of the fictitious resistance may be roughly expressed by saying that it varies inversely as the frequency. From equation (I) it follows that

$$
\theta_{2}-\theta_{1}=p\left(C_{1} r_{1}-C_{2} r_{2}\right)
$$

which determines the phase difference for the series resistance method.

(c) Advantages and Disadvantages of Each Method

The series resistance method is very useful where two condensers of very different quality are to be compared, since one may use for the compensating resistance $r$ a resistance box having a wide range of resistances. However, it has the disadvantage of introducing the capacity of this compensating resistance into the measured capacity. In the case of fairly large capacities, $r$ may be kept small, and the error introduced is not serious; but in the case of very small capacities the error may be very appreciable.

The chief difficulties in connection with the series inductance method are the correction factor and the difficulty of finding a variable inductance having a suitable range for any set of measurements. As already indicated, the correction factor in the case of condensers such as we are considering can be neglected, provided suitable inductances are used. By making both $L_{3}$ and $L_{4}$ variable inductances, the second difficulty is partially overcome. In any case with condensers of good quality and a fair range of inductances from which to choose, the difficulty is more apparent than real.

It will be seen that for the kind of condensers used in this investigation the series inductance method is to be preferred, and it has been used almost exclusively. However, in either case a substitution method will give better results than a direct comparison of capacities. In this case $C_{2}$ is an auxiliary condenser, 
preferably of about the same value as the condensers to be compared. The standard condenser $C_{1}$ is placed in the bridge and a reading taken. It is then replaced by the unknown condenser $C_{1}{ }^{\prime}$ and a second reading taken. From these readings the capacity of $C_{1}{ }^{\prime}$, in terms of $C_{1}$ is readily computed.

\section{(d) A Direct=Reading Capacity Bridge}

In order to make the bridge direct reading, the following method of procedure was devised. With $C_{1}$ in the bridge, $R_{4}$ is set so that its resistance is some decimal multiple of the numerical value of $C_{1}$; then $R_{3}$ and $L_{4}$ are varied until a balance is obtained.

Then $C_{1}=R_{4} \frac{C_{2}}{R_{3}}$

Now replace $C_{1}$ by the unknown condenser $C_{1}{ }^{\prime}$ and adjust $R_{4}$ and $L_{4}$ for a balance. Let this value of $R_{4}$ be $R_{4}{ }^{\prime}$. Then

$$
C_{1}{ }^{\prime}=R_{4}{ }^{\prime} \frac{C_{2}}{R_{3}}=R_{4}{ }^{\prime} \frac{C_{1}}{R_{4}}
$$

But since $C_{1}$ numerically equals $R_{4}, C_{1}{ }^{\prime}$ numerically equals $R_{4}{ }^{\prime}$.

\section{(e) The Choice of Resistance}

In selecting resistances for use in the arms $R_{3}$ and $R_{4}$, three things must be considered, viz, the effect of errors in the adjustment of the resistance coils on the measured value of the capacity, the permissible current through the coils, and the effect of residual inductances and capacities in the coils. In using the substitution method the errors in the adjustment of $R_{3}$ do not enter, and those of $R_{4}$ enter only in so far as $\frac{R_{4}{ }^{\prime}}{R_{4}}=\mathrm{I}+\frac{\Delta R_{4}}{R_{4}}$ is in error. With capacities of approximately the same value and a well adjusted resistance box, any correction on this account is negligible. The effect of residual inductances and capacities in the coils is to introduce an error in the value of $L_{4}-L_{4}{ }^{\prime}$ by the amount that the effective inductance of $R_{4}^{\prime}$ differs from that of $R_{4}$. In ordinary cases this is negligible, but at high frequencies it becomes so important that accurate measurements of the phase difference are often impossible. 
The permissible current through any coil will depend on its resistance as well as on the readiness with which it conducts away the heat generated. With coils of the ordinary size, immersed in oil, the current should be such that the expenditure of power in any one coil is not over one watt. With even moderate frequencies and voltages and a medium-sized condenser it is not permissible to use a Iooo-ohm coil, and at high frequencies it is often desirable to avoid the use of a roo-ohm coil. Hence, if measurements are to be made to a part in a hundred thousand, it is necessary to have some means of varying $R_{4}$ by steps of a thousandth of an ohm. For this purpose a specially designed resistance box was constructed. It consists of three coils in series permanently connected between the terminals of the box, around each of which there is arranged a variable shunt. These shunts are of such values that each step on the first dial varies the resistance between the terminals by a tenth of an ohm, each step on the second dial varies the resistance by a hundredth of an ohm, and on the third dial each step varies the resistance by a thousandth of an ohm. The resistance when all of the dials are on zero is ro ohms. The advantage of this over a slide wire is that all the contacts are in the shunt circuits which have comparatively high resistance, so that any variation of contact resistance will produce a negligible effect.

\section{A. C. COMPARISON OF A CAPACITY WITH AN INDUCTANCE}

The Anderson bridge method has been used whenever it has been necessary to compare a capacity with an inductance by alternating current. This has been fully described, ${ }^{10}$ and needs only be mentioned here. Ordinarily an inductance is measured in terms of a capacity, but once the value of an inductance is determined, the process may be reversed. This may be desirable in investigating the constancy of a condenser, since an inductance wound on marble is a very permanent standard. 


\section{DIRECT CURRENT COMPARISONS OF CAPACITY}

(a) The Differential Galvanometer Method

For direct current comparisons of capacity two methods have been tried, the differential galvanometer method and the method of mixtures. In the differential galvanometer method two condensers are charged with approximately equal quantities of electricity, then discharged simultaneously through the two coils of a differential galvanometer. If the discharge does not begin at practically the same instant through both coils there will be a slight movement of the galvanometer mirror, so that it is very difficult to tell whether or not there is a true deflection. Moreover, in comparing two condensers having different absorptions, the rate at which the absorbed charge comes out of the two condensers may be quite different, so that there may be a slight deflection even though the total quantity which passes through each of the coils is the same. For these and other reasons the differential galvanometer method for the comparison of capacities has been abandoned.

\section{(b) The Method of Mixtures}

In the method of mixtures (see Fig. 4) the battery, $B$, maintains a current in the resistances $R_{1}$ and $R_{2}$. On closing the key $K_{1}$ the condensers $C_{1}$ and $C_{2}$ are charged to the potential differences between the terminals of $R_{1}$ and $R_{2}$, respectively. When the key $K_{1}$ is released, the condensers discharge against each other and any free charge which remains is discharged through the galvanometer on pressing the key $K_{2}$. A small resistance, $r$, is inserted to prevent oscillatory charge or discharge. If $C_{1}$ and $C_{2}$ are air condensers, by adjusting $R_{1}$ or $R_{2}$ and repeating the operation, a point is soon reached where there is no deflection of the galvanometer. Then

$$
R_{1} C_{1}=R_{2} C_{2}
$$

By using the substitution method, this arrangement can be made direct reading in the same manner as with the alternating current bridges.

With condensers which show absorption the apparent capacity will depend upon the length of the charging period, the length of time of mixing before discharging through the galvanometer, and 
on the previous history of the condenser. If a condenser is charged to a high voltage for some time, an appreciable absorbed charge will continue to come out of it for a long time thereafter. To completely discharge a condenser is often very difficult, but in the case of a mica condenser it is usually possible to discharge it to such a point that the residual charge which appears in the first second is negligible.

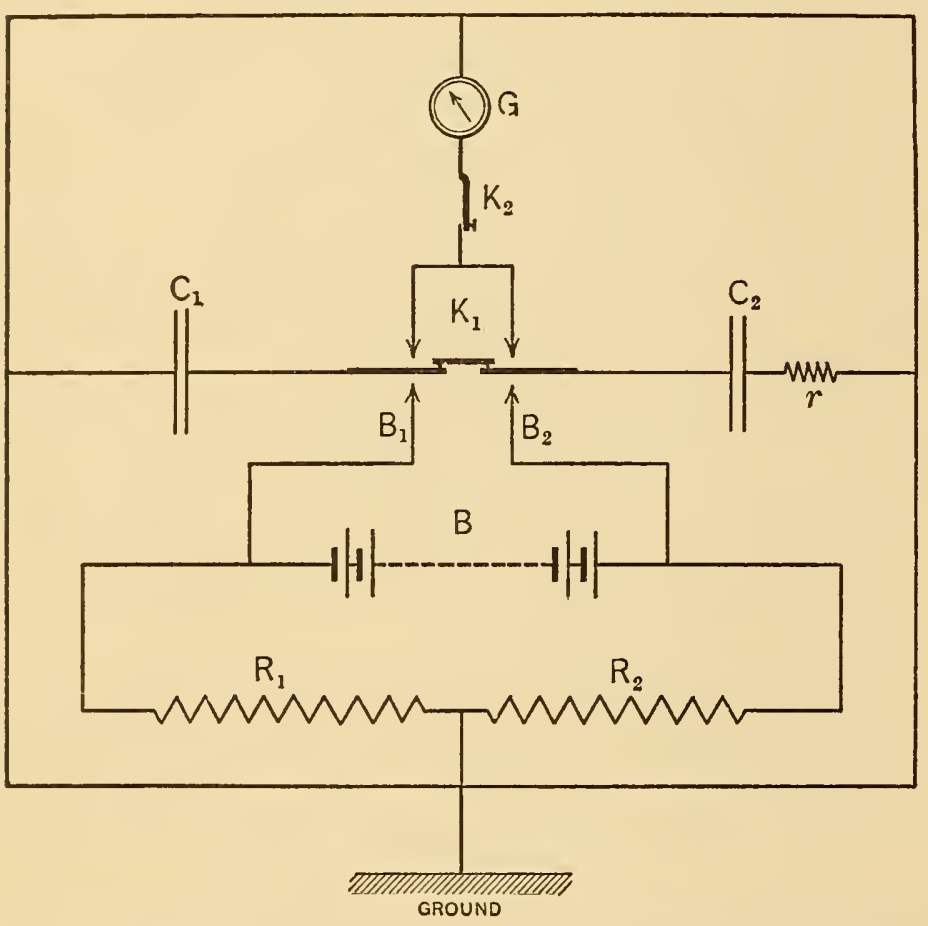

NOMENCLATURE.

$\mathrm{R}_{1}$ and $\mathrm{R}_{2}$ are resistances. $\mathrm{C}_{1}$ and $\mathrm{C}_{2}$ are capacities. $B$ is a battery. $\mathrm{G}$ is a galvanometer. $\mathrm{K}_{1}$ is a double charge and discharge key. $\mathrm{K}_{2}$ is a single key.

Fig. 4.-Diagram to show the method of comparing capacities by the method of mixtures

Having discharged the condensers, the method of procedure is to charge the condensers for a definite time by depressing the key $K_{1}$, then to release $K_{1}$, and after a certain definite time to close the key $K_{2}$. If there is a deflection of the galvanometer mirror, adjust the resistances, thoroughly discharge the condensers, and repeat the process. Continue until there is no deflection of the galvanometer. This gives the Acyclic Capacity under the given conditions. It should be noticed that the time of contact of $K_{2}$ must be very short, since if the absorbed charges are coming out from the condensers at different rates, there will be a current through the galvanometer which may produce a deflection, even though there was no free charge at the instant of closing the key. 
The above method will give very accurate results, but is very slow in operation. A method of improving it in this respect is to charge and discharge the condensers periodically. The capacity determined in this way we will call the Cyclic Capacity. Then in each discharge the total residual charge consists of portions from each of a number of previous charges. Hence the cyclic capacity is larger than the acyclic capacity, the time of charge and discharge being the same in both cases.

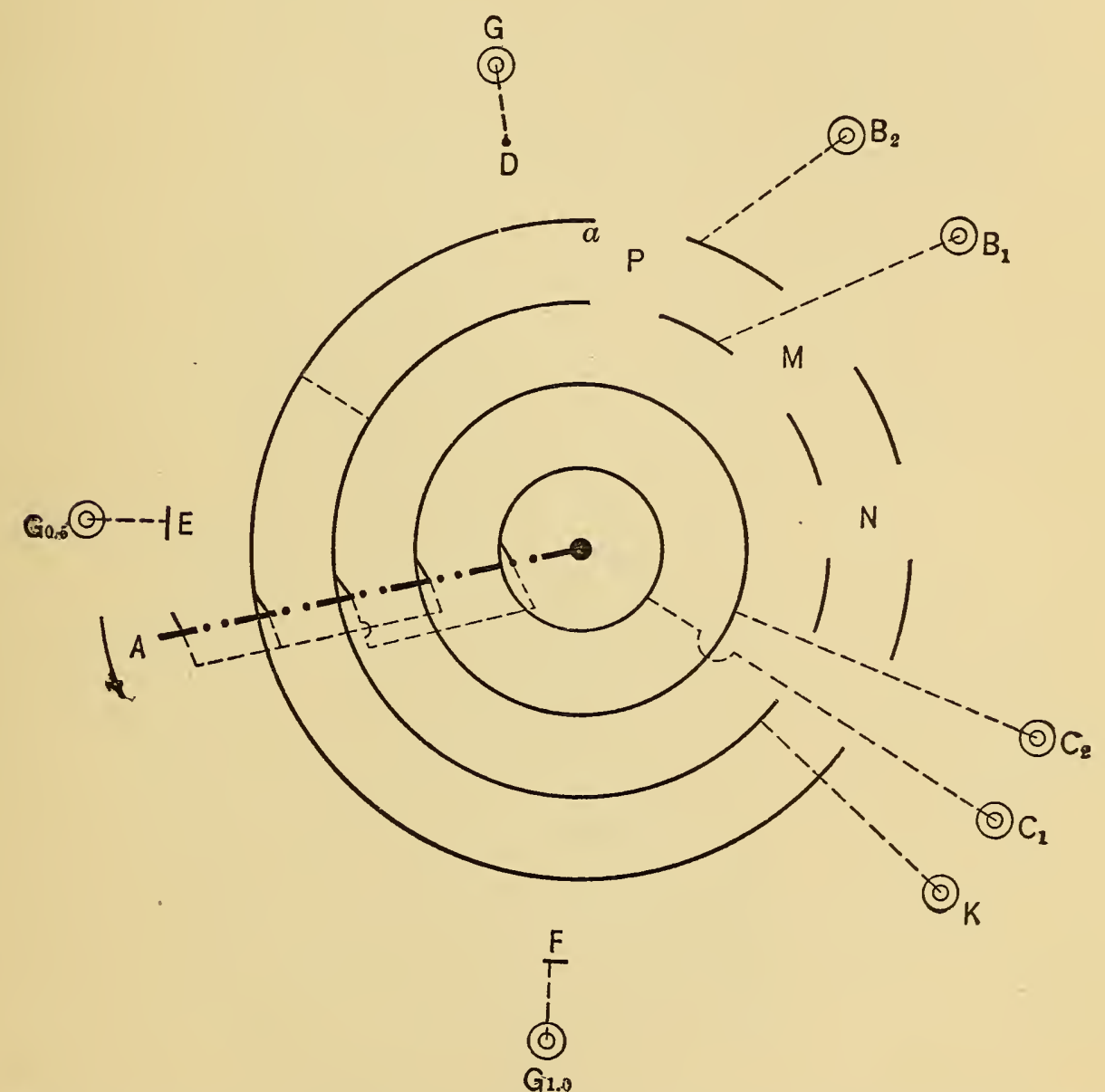

Fig. 5.-Diagram of apparatus for charging and discharging condensers in the method of mixtures

To accomplish the periodic charging and discharging of the condenser, as well as to make the time of charge and discharge of the condensers as definite as possible when the acyclic method is used, the apparatus shown in Figs. 5 and 6 was constructed. This apparatus merely replaces the keys $K_{1}$ and $K_{2}$ in the preceding diagram. It consists of a hard rubber base on which are mounted

$$
48848^{\circ}-\mathrm{ro}-2
$$


portions of five brass rings. Above this rotates a hard rubber arm $A$ which carries five brushes that bear upon the rings. This arm is driven by a motor so geared that the arm makes one revolution every two seconds. With a variable resistance in the armature circuit of the motor, and a clock ticking seconds, the adjustment of the speed of the motor can easily be made with the necessary accuracy. The inside condenser terminals are connected to the binding posts $C_{1}$ and $C_{2}$, and the battery terminals to the posts $B_{1}$ and $B_{2}$. If the acyclic capacity is desired it is necessary to insert single contact keys between the posts $B_{1}$ and $B_{2}$ on the one hand and the battery terminals on the other. The galvanometer may be connected to any of the posts marked $G$. If connected to the post $G_{0.5}$ the length of mixing is 0.5 second. If connected to the post $G_{1 \cdot 0}$ the length of mixing is r.o second. The point $D$ connected to the post, $G$, can be moved along the circumference of the circle, so that the time of mixing can be varied from o. I second to o.or second, or even less. When these short times are used, the time of mixing is determined electrically, as will be explained later. The method of connecting the brushes is indicated by the dotted lines in front of the $\operatorname{arm} A$.

Let us suppose the galvanometer connected to $G_{1 \cdot 0}$, and the arm $A$ rotating in the direction indicated by the arrow. When the arm passes the opening $M$ and the brushes touch the brass pieces beyond, the condensers are charged almost instantly, and remain charged while passing across $P$, thus making the total time of charge 0.2 second. The condensers are discharged when the brushes touch the pieces beyond $P$, there remaining the quantity by which the free charges of $C_{1}$ and $C_{2}$ differ. From this time until the galvanometer brush touches $F$ which is connected to $G_{1 \cdot 0}, C_{1}$ and $C_{2}$ are giving up their absorbed charges. If the algebraic sum of these absorbed charges is just equal to the residual of the free charge, there will be no discharge through the galvanometer. In this way the apparent capacity of $C_{1}$ under the given conditions is determined, provided $C_{2}$ is known.

Small brass pieces are provided which fill the gaps $M$ and $N$ in the third and fourth rings, so that the time of charge may be made 0.2 second, 0.4 second, or 0.6 second. A brass piece is also provided which may be connected to $D$, this extending the arc from $D$ to beyond the diameter which passes through $a$. This is 


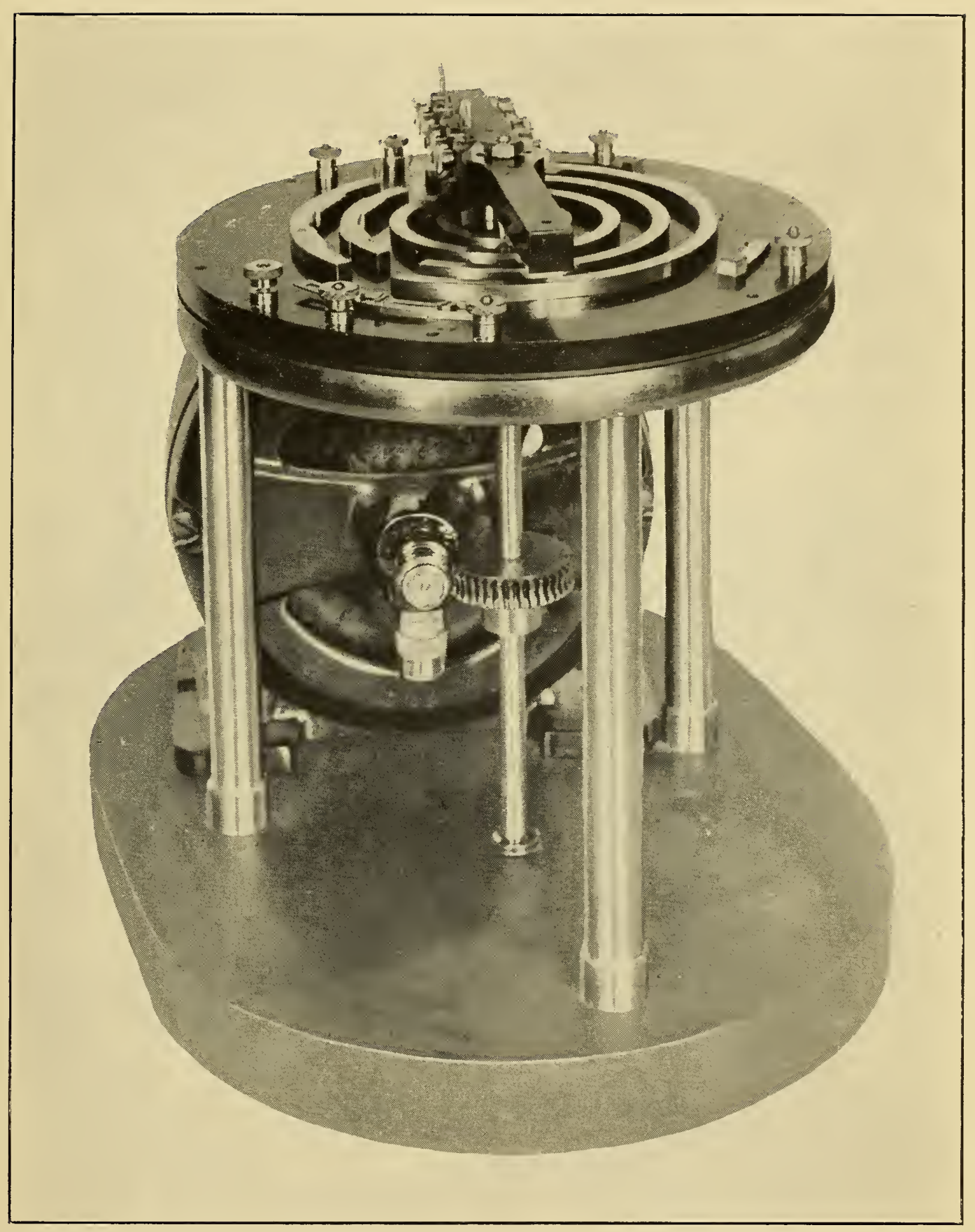

Fig. 6 - Apparatus for charging and discharging condensers in the method of mixtures 
for determining the time of mixing and is used in the following manner, when it is necessary to employ apparatus not regularly used in this set-up. A battery whose emf is $E$ is connected to a condenser of capacity $C$, and the charge which collects on the condenser is discharged through a long-period ballistic galvanometer giving a deflection $d$. The same battery and galvanometer are then connected in a circuit with a high resistance, a single contact key, and the posts $K-G$ of this apparatus. With the arm rotating, if the key is closed a current will flow from the time the fourth brush touches the ring beyond $P$ until the fifth brush leaves $D$. This will give a ballistic throw to the galvanometer. Adjust $R$ until there is the same deflection $d$ as when the condenser was discharged. Then

$$
E C=\frac{E t}{R} \text { or } t=R C
$$

If the third brush touches the ring beyond $P$ after the fourth brush has touched its ring, the connections on the rotating arm must be changed so that the third brush is connected to the fifth. This is necessary, since the discharge of the condensers does not begin until the last of the two brushes touches its ring on passing $P$.

\section{METHODS OF "STEPPING UP"}

As stated previously, the apparent capacity of a mica condenser under any condition is determined by comparing it with an air condenser of the same nominal value, provided such a condenser is available. But as o.I $\mathrm{mf}$ is the largest air condenser in this laboratory, and as it is often necessary to measure values of 0.5 or I.o mf, some means of "stepping up" from lower to higher values is necessary.

If a mica condenser is constructed so that both of the outside sheets of tin foil are connected to the same terminal, and if this terminal is connected to earth during the measurement, then the capacity is practically independent of its surroundings. This terminal is called the outside or $A$ terminal. In A. C. work, using an insulated system, the mean value of the potential of either terminal of the machine is sensibly the earth potential, so that in A. C. bridges the outside terminal of the condenser is connected directly to one terminal of the machine. Such con- 
densers may be used additively, and their capacity when in parallel is the sum of their individual capacities. They can not be connected in series where high precision is desired, since it is impossible to so arrange them that the outside plates of both condensers will be at the earth potential, though with alternating current the error is very small.

To determine the apparent capacity of a microfarad using a tenth microfarad air condenser, there are several possible procedures. One of the simplest is to compare five tenth-microfarad mica condensers with the air condenser, then compare their sum with a half-microfarad, and take the sum of the six condensers as the standard microfarad.

In the above discussion it has been assumed that each condenser has separate terminals, so that in connecting the condensers together no appreciable capacity will be introduced on this account. With condensers having large terminal blocks the capacity between these blocks often introduces a very troublesome correction.

\section{MEASUREMENT OF ABSORPTION}

It will be shown later that there are two factors which should be considered in determining the absorption of a condenser. One is the rate at which the absorbed charge reappears and the other is the value of the total absorbed charge. These quantities are very difficult to measure. Hence the following simple procedure has been adopted as giving some knowledge of the relative qualities of different condensers.

The condenser is charged for one second, insulated for one second, short-circuited for one second, then insulated for thirty seconds, at the end of which time it is instantaneously discharged through a ballistic galvanometer. It is then insulated for thirty seconds and again discharged through the galvanometer. This is continued until five residuals have been measured. The sum of these deflections multiplied by the constant of the ballistic galvanometer gives the measured portion of the absorbed charge. The product of the capacity of the condenser by the charging voltage gives the free charge. The absorption is taken as the ratio of the measured portion of the absorbed charge to the free charge. 


\section{EXTERNAL CONDITIONS AFFECTING THE CAPACITY}

\section{THE TEMPERATURE COEFFICIENT}

Each apparent capacity of a condenser has its own temperature coefficient. In other words, the temperature coefficient of the capacity measured with Iooo cycles is different from that measured with Ioo cycles, and both of these are different from that obtained by measurements with direct current. It will be shown later that the difference between the different apparent capacities is due to absorption, and that the true ${ }^{11}$ or geometric capacity would be obtained either if the condenser could be instantly discharged when being measured by direct current, or if it could be measured with alternating currect of infinite frequency. The geometic capacity can not be measured directly, so that the true temperature coefficient of the capacity is not easily determined. However, the capacity at roo cycles is but little different from the geometric capacity, so that in the following it is assumed, unless otherwise stated, that the temperature coefficient at 100 cycles is the true temperature coefficient of the capacity.

There is a wide variation in the temperature coefficient of mica condensers. With silvered-mica condensers the temperature coefficient is small and positive. With the ordinary mica condensers, where paraffin forms a part of the dielectric, it is usually negative and may be as large at o.I per cent per degree, though in most cases it is very much smaller. In seeking the cause for this wide variation, attention was attracted to two $0.5-\mathrm{mf}$ condensers in the same case, one of which had a temperature coefficient of 6 parts in a hundred-thousand per degree, while the other had a temperature coefficient of 32 parts. An examination showed that the condenser with the larger temperature coefficient was much thicker than the other condenser. At the suggestion of Dr. Rosa, this condenser was placed in molten paraffin, and when thoroughly warmed through, was compressed in a vise and left until cold. The result was that the capacity was increased over $5^{\circ}$ per cent, while the temperature coefficient was reduced to only one part in a hundred thousand per degree. This same treatment was then tried on a number of condensers, and in all cases the temperature coefficient became less. In some it actually changed

${ }^{11}$ A Zeleny, Phys. Rev. 22, p. 65 ; 1906. 
sign and became positive. Of course following this treatment it is necessary to readjust the capacity if a definite value is desired. It should also be remarked that this treatment always affected the phase difference, sometimes favorably, sometimes unfavorably. Table III gives some of the results.

TABLE III

\begin{tabular}{rr|c|c|c|c}
\hline \multirow{2}{*}{ Condenser } & \multicolumn{2}{|c|}{ Temperature coefficient in parts in 100,000 } & \multicolumn{2}{|c}{ Phase difference } \\
\cline { 2 - 3 } & Before treatment & After treatment & Before treatment & After treatment \\
\cline { 3 - 5 } No. 1 & -14.5 & -3.7 & $4^{\prime} 20^{\prime \prime}$ & $6^{\prime} 30^{\prime \prime}$ \\
& 2 & -9.1 & +0.5 & $8^{\prime} 50^{\prime \prime}$ & $13^{\prime} 25^{\prime \prime}$ \\
3 & -14.0 & -5.0 & $5^{\prime} 20^{\prime \prime}$ & $7^{\prime} 10^{\prime \prime}$ \\
5 & -7.0 & -5.0 & $3^{\prime} 20^{\prime \prime}$ & $2^{\prime} 45^{\prime \prime}$ \\
10 & -10.7 & +1.8 & $5^{\prime} 30^{\prime \prime}$ & $4^{\prime} 35^{\prime \prime}$ \\
6 & -6.4 & -1.5 & $3^{\prime} 25^{\prime \prime}$ & $4^{\prime} 10^{\prime \prime}$ \\
$4460 \mathrm{R}$ & -31.0 & -1.0 & $2^{\prime} 20^{\prime \prime}$ & $2^{\prime} 0^{\prime \prime}$ \\
$7763_{1}$ & -11.8 & -4.5 & $2^{\prime} 5^{\prime \prime}$ & $1^{\prime} 20^{\prime \prime}$ \\
$7763_{2}$ & -12.6 & -2.0 & $2^{\prime} 40^{\prime \prime}$ & $2^{\prime} 00^{\prime \prime}$ \\
$7763_{3}$ & -3.6 & -1.0 & $1^{\prime} 40^{\prime \prime}$ & $2^{\prime} 5^{\prime \prime}$ \\
\hline
\end{tabular}

It is desirable to have the temperature coefficient of any standard as low as possible, but this is especially true with a condenser, since its thermal conductivity is so poor. With the best of these condensers an error of several tenths of a degree in the measurement of the temperature, will not introduce an error of a part in a hundred thousand in the capacity.

The experiments of Rosa and Grover show that for condensers with a high melting point paraffin the temperature coefficient is approximately a constant below $30^{\circ} \mathrm{C}$. Experiments were now undertaken to determine the exact form of the capacity-temperature curve, and at the same time to see how the temperature coefficient is affected by the method employed in measuring the capacity. The condensers were placed in a case in which the temperature could be maintained constant at any desired temperature to $0 . \mathrm{I}^{\circ} \mathrm{C}$ by means of a vapor pressure thermostat. The reference condensers were in another case which was maintained at $25^{\circ} \mathrm{C}$ throughout the experiments by a similar thermostat. Each of the condensers had a capacity which did not 
differ greatly from o.I mf. This value was chosen so that the condensers could be compared directly with an air condenser. At the beginning of the experiments a careful determination was made of the apparent capacity at Ioo cycles of the two reference condensers. The mean of these condensers was then assumed constant, and the value of the air condenser at any time was determined by comparing it with the reference condensers by means of A. C. of Ioo cycles. The relative values of the two reference condensers changed slightly during the course of the experiments, but a careful absolute measurement failed to show that the mean value had changed appreciably. The results are shown in the accompanying curves. (Figs. 7 to I2.) All of these curves are plotted to the same scale, so that the relative behavior of the different condensers is easily followed. Not only is there indicated the change with temperature of the capacity at different frequencies of A. C. and different times of discharge with D. C., but also the change with temperature of the phase difference, of the absorption, and in some cases of the insulation resistance.

Condensers No. 3 and No. $547 \mathrm{IB}$ are of American make, and have metal clamps. The o. $\mathrm{Imf}$ section of $547 \mathrm{IB}$ is a very satisfactory condenser. The temperature coefficient under all conditions is small and the change of capacity with frequency is not large; also the phase difference and absorption are small. The So.I mf section of $547 \mathrm{I}$ B behaves very well with A. C. Its change with frequency is but little more than is found in the best condensers and its phase difference is not large in any case. However, with direct current the change of capacity with the length of discharge is very marked, and the absorption is also large. The temperature coefficient of No. 3 is not large, but otherwise the behavior is not satisfactory on either A. C. or D. C. The curves are given as an example of what may be expected of a poor mica condenser.

Condenser 1038 is of German manufacture. It is one of the oldest condensers in this laboratory, and has wooden clamps. The section here shown is very similar to the other sections which have been examined. All of the sections have a large negative temperature coefficient on A. C., but otherwise behave very well on both A. C. and D. C. Condenser No. 20 was manufactured by Siemens and Halske according to specifications furnished by this 
bureau. A high melting point paraffin was used in its construction, and it is compressed between heavy metal clamps. The behavior with A. C. is especially good, the phase difference at I 200 cycles being so small that it could not be satisfactorily measured. With D. C. it does not make quite so good a showing, but is still among the very best. There is also shown the curves for a silvered mica condenser. However, as will be pointed out later, there are factors influencing the behavior in this condenser which are not operative with the others, so that conclusions which may be drawn respecting the other condensers are not applicable to this one. However, among all the condensers examined, this is the only one that needs to be treated as an exception.

The apparent capacity at $\mathrm{r} 200$ cycles is very little different from the geometric capacity, as will be shown later. Hence any conclusions drawn from the values of the capacity at I 200 cycles will be applicable to the geometric capacity. The figures show that the capacity-temperature curve at $\mathrm{r} 200$ cycles is very nearly a straight line within the range investigated ( 5 to $30^{\circ} \mathrm{C}$ ). In three cases the curvature, if any, is too small to be detected. In four cases the curves are convex, upward; i. e., there is an algebraic decrease of the temperature coefficient with increasing temperature. The magnitude of these changes will be seen from Table IV. In no case is the change sufficiently large to warrant the application of a parabolic formula.

\section{TABLE IV}

Temperature Coefficient at 1200 Cycles in Various Temperature Intervals

\begin{tabular}{l|c|c|c}
\hline \multicolumn{1}{c|}{ Condenser } & \multicolumn{3}{|c}{$\begin{array}{c}\text { Temperature coefficient in parts per hun- } \\
\text { dred thousand per degree }\end{array}$} \\
\hline & $15^{\circ}-20^{\circ}$ & $20^{\circ}-25^{\circ}$ & $25^{\circ}-30^{\circ}$ \\
\hline $5471 B 0.1$ section & -3 & -8 & -11 \\
$\Sigma 0.1$ section & -5 & -5 & -5 \\
10380.1 section & -15 & -22 & -22 \\
10380.2 and 0.2 in series & -19 & -23 & -27 \\
No. 20 & -5 & -5 & -5 \\
Nos. 13 and 14 in series & +3 & +3 & +3 \\
No. 3 & -5 & -10 & -16 \\
\hline
\end{tabular}




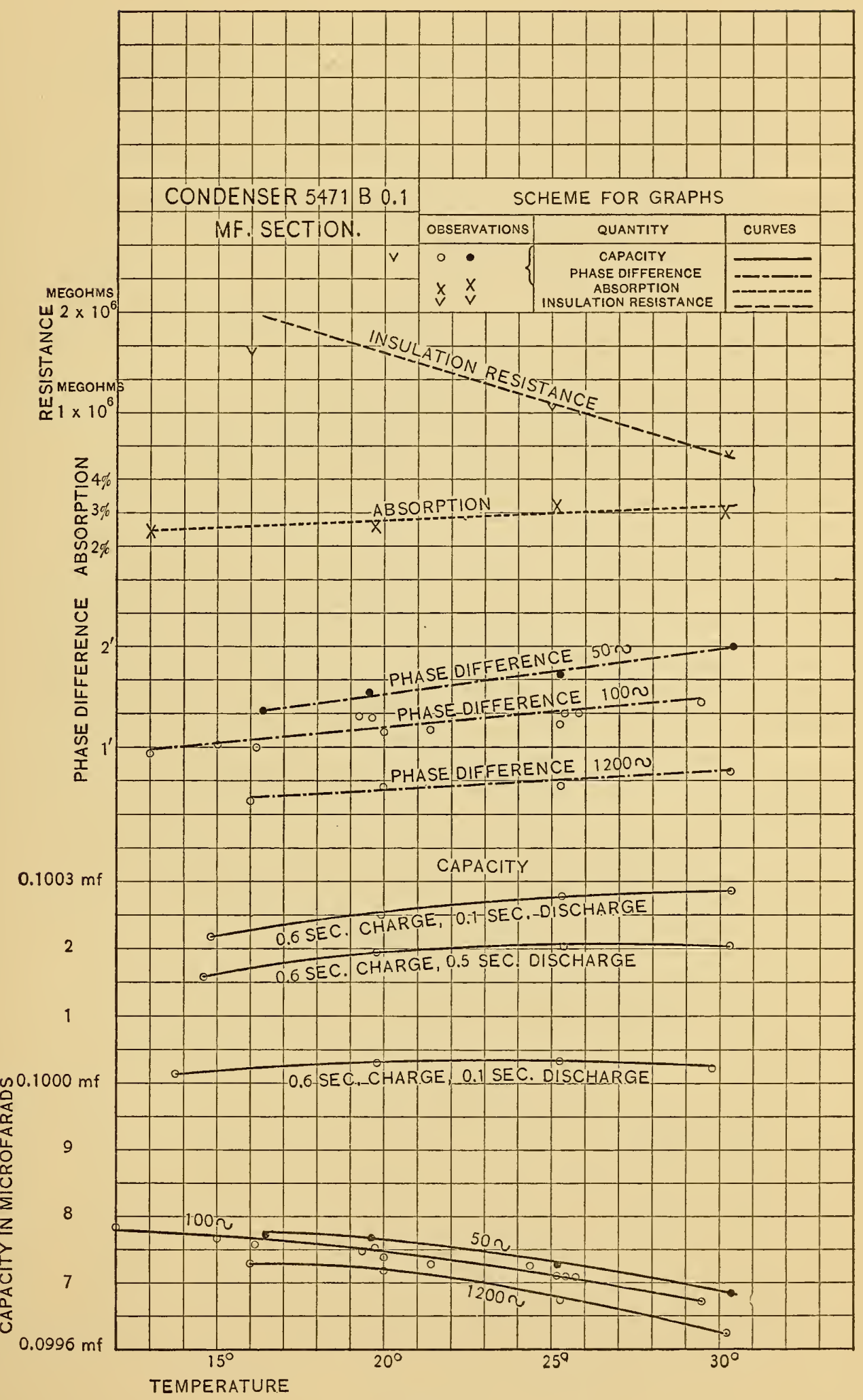

Fig. 7 


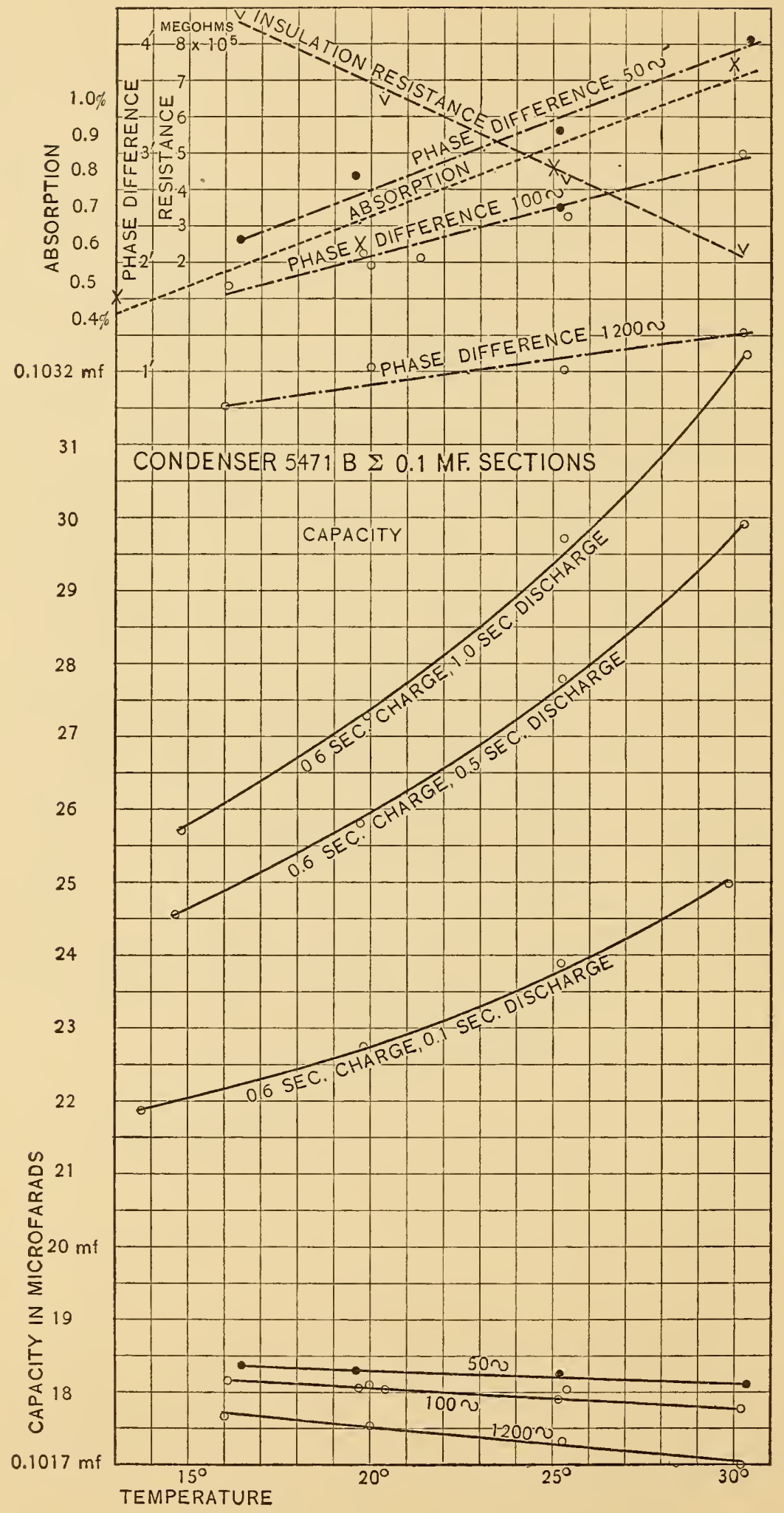

Fig. 8 


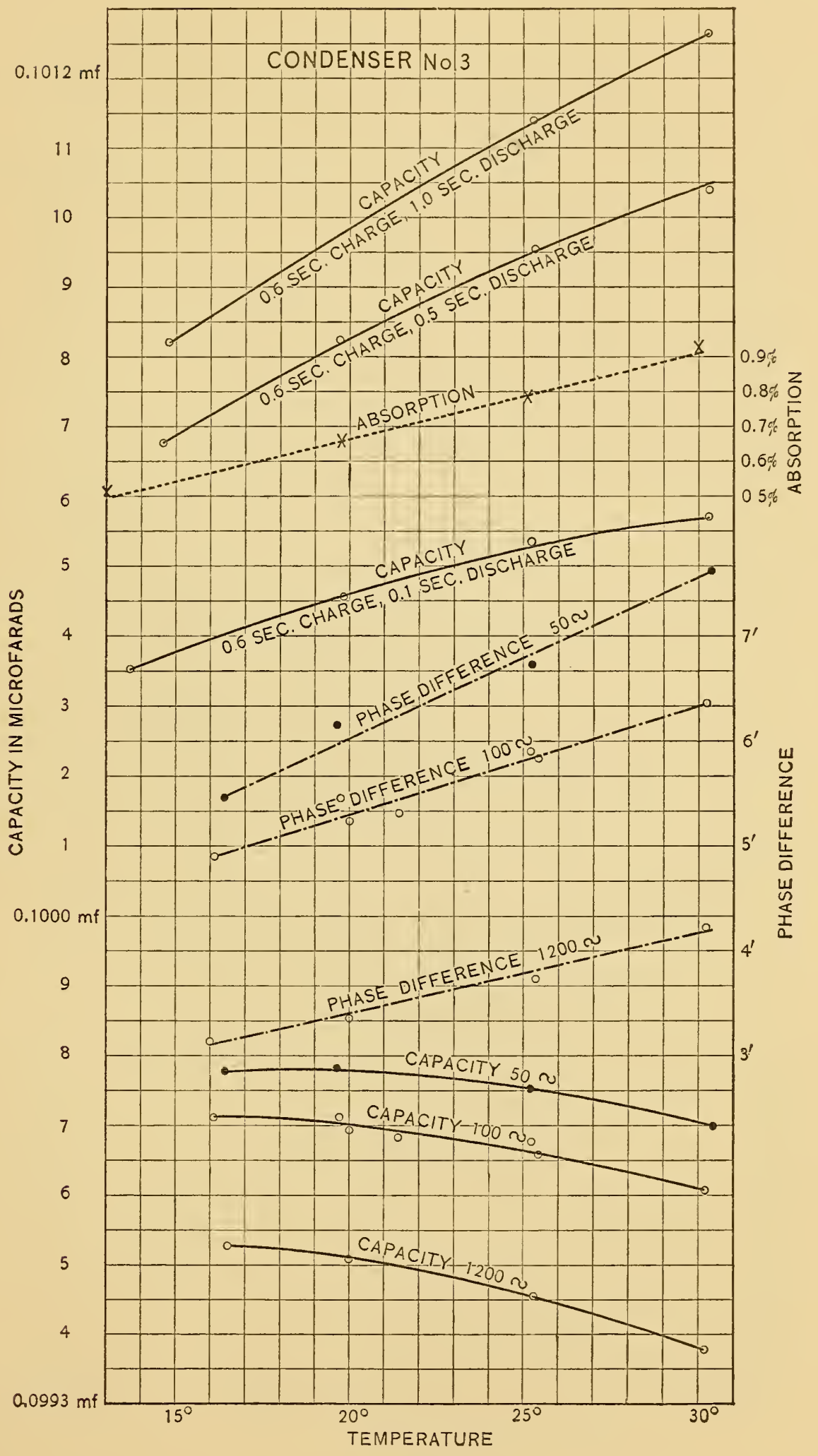

Fig. 9 


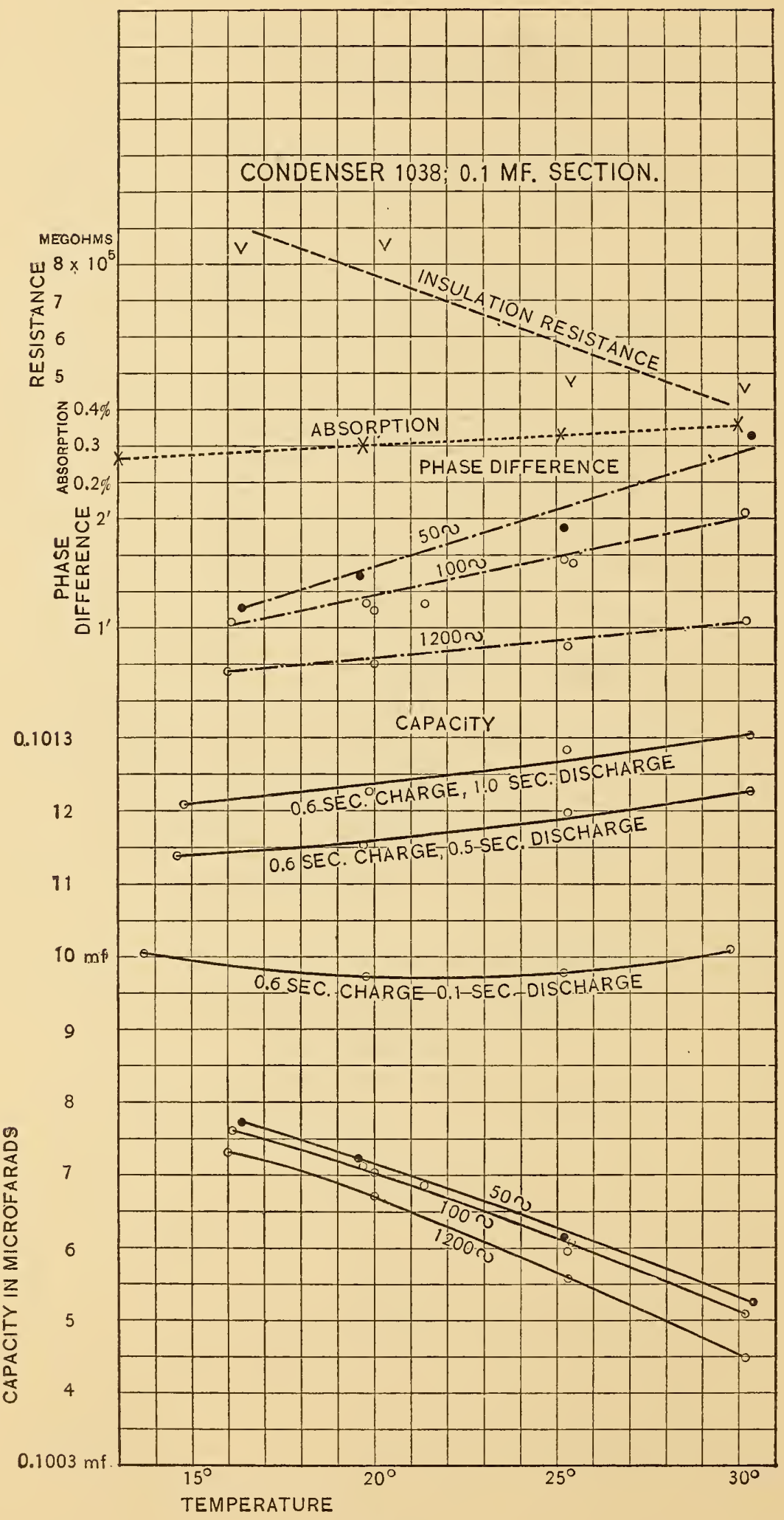

Fig. 10 


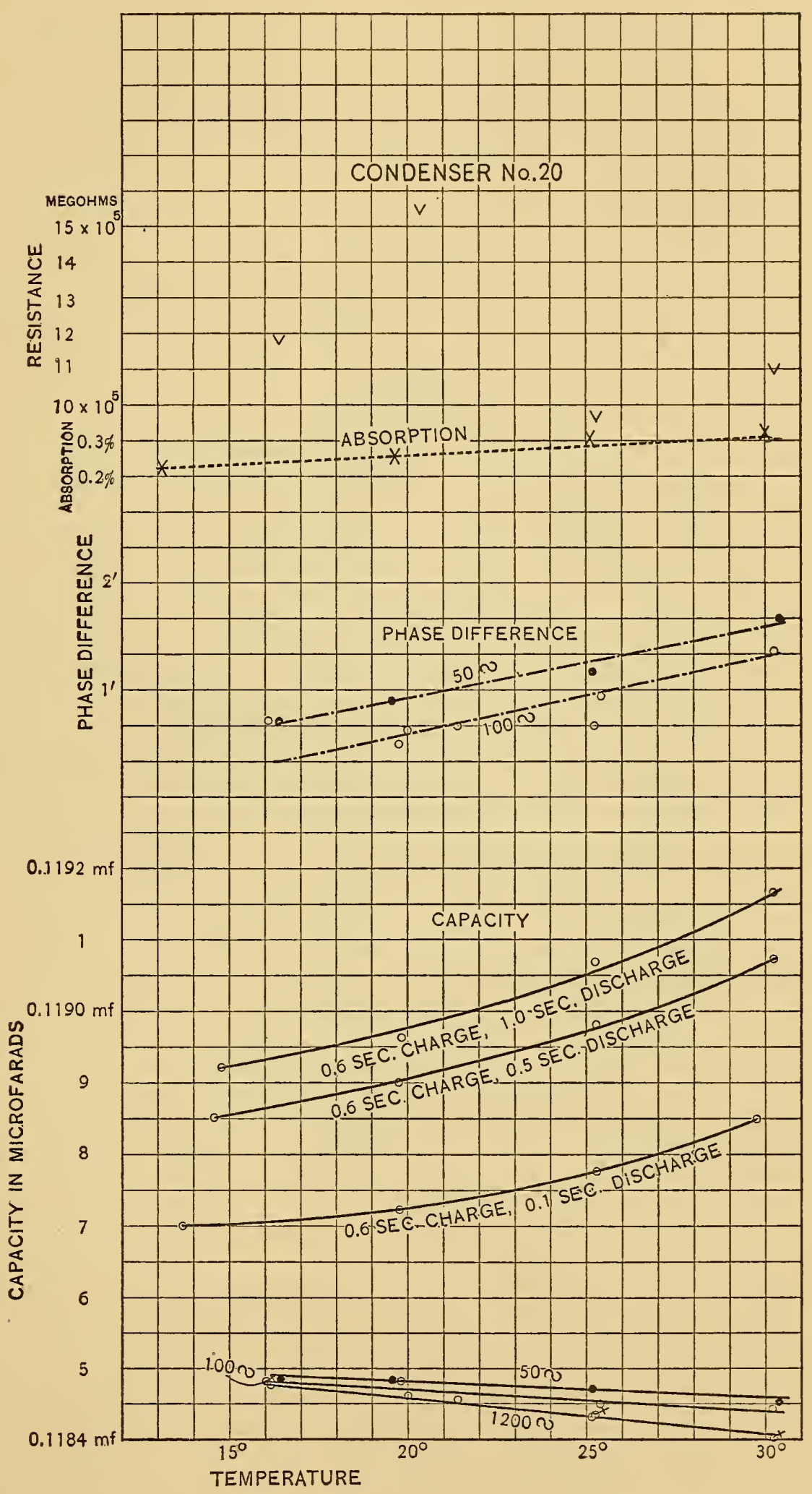

Fig. 11 
[Vol. 6, No. 4 .

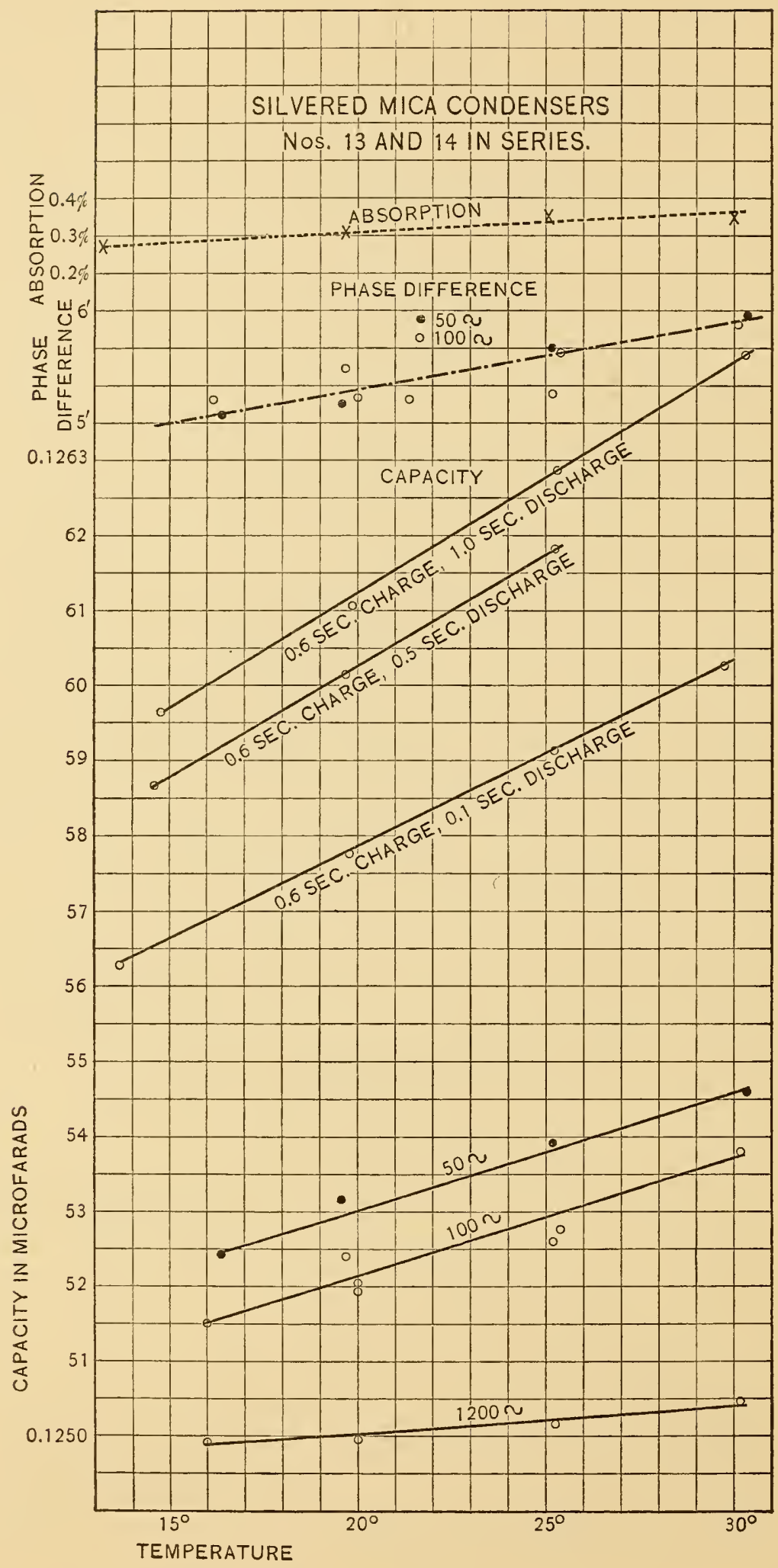

Fig. 12 
In going to lower frequencies there is always an algebraic increase in the mean temperature coefficient of the apparent capacity, but over a wide range of frequencies this is not large. However, with the relatively long times of discharge used in the D. C. measurements there is often a very decided change in the temperature coefficient. The effect may be expressed qualitatively by saying that the temperature coefficient of the apparent capacity increases algebraically as the period increases. This is well shown in Table V:

\section{TABLE V}

Mean Temperature Coefficient at Different Frequencies

\begin{tabular}{|c|c|c|c|c|c|c|}
\hline \multirow[b]{2}{*}{ Condenser } & \multicolumn{3}{|c|}{ A. C. } & \multicolumn{3}{|c|}{ D. C. 0.6 Sec. Charge } \\
\hline & $1200 \sim$ & $100 \sim$ & $50 \sim$ & $\begin{array}{l}0.1 \text { Sec. } \\
\text { Dis. }\end{array}$ & $\begin{array}{l}0.5 \mathrm{Sec} . \\
\text { Dis. }\end{array}$ & $\begin{array}{l}\text { 1.0 Sec. } \\
\text { Dis. }\end{array}$ \\
\hline 5471B 0.1 section & -7 & -6 & -6 & +1 & +3 & +4 \\
\hline$\Sigma 0.1 \quad$ “ & -5 & -3 & -2 & +17 & +35 & +42 \\
\hline 10380.1 & -20 & -18 & -17 & 0 & +6 & +6 \\
\hline 10380.2 and 0.2 in series & -23 & -22 & -21 & -2 & +3 & +5 \\
\hline No. 20 & -5 & -3 & -2 & +10 & +14 & +16 \\
\hline Nos. 13 and 14 in series & +3 & +15 & +15 & +25 & +30 & +31 \\
\hline No. 3 & -10 & -7 & -5 & +13 & +23 & +28 \\
\hline
\end{tabular}

With the D. C. measurements the capacity-temperature curve is often not even approximately a straight line. However, as there seems to be no simple way of connecting the equations of the different curves, and as it is scarcely desirable to compute a parabolic form for each apparent capacity, it is better to refer directly to the curves when calculations from the mean temperature coefficient are not sufficiently accurate.

The explanation of the above phenomena is to be found in a consideration of the absorption of the condensers. The increase of the absorption with increasing temperature is very large, in some cases being as much as roo per cent in the interval considered. Moreover there may be a change in the form of the absorption curve as the temperature is changed. Since it is due to absorption that there is a difference in the apparent capacities of a condenser, any increase in the absorption will increase this 
difference. Hence we must expect that there will be an algebraic increase in the temperature coefficient with increasing period due to absorption, and this agrees with experiment. It is not so easy to draw conclusions as to the effect of the change in shape of the absorption curve. However, it will be noticed that the changes in the shape of the $\mathrm{D}$. C. capacity curves are in all cases progressive changes as the length of discharge is increased. Any change of this sort can of course be explained by a change in the shape of the absorption curve.

It is well to notice that in all cases there is an increase in the phase difference with increasing temperature, and that the lower the frequency the greater is this increase. Also the insulation resistance decreases very markedly on going to higher temperatures. It is therefore very evident that a mica condenser gives the most satisfactory results at relatively low temperatures.

\section{THE PRESSURE COEFFICIENT}

Since a condenser is normally subjected to the changes of the atmospheric pressure, it seemed desirable to see if there is a change in capacity due to this cause. Accordingly a condenser was placed under a bell-jar through which leads were passed, and the air partially exhausted. Measurements of the capacity before and after exhaustion showed not only that there is a decrease in the capacity when the pressure is decreased, but also that some time is required after a change in pressure before an equilibrium condition is reached, during which time there is a continuous change in the capacity. An idea of the rate at which the capacity changes, in the case of a condenser which shows a very marked change with pressure, can be obtained from Table VI. In another experiment it was found that some very good condensers which were placed in a desiccator and the pressure reduced to I mm of mercury did not reach equilibrium for several days.

In order to see if this after effect is not negligible when there is only a small change in pressure, a number of condensers were measured, first at atmospheric pressure, then at a pressure $6 \mathrm{~cm}$ less than atmospheric pressure and finally at atmospheric pressure. Any after effect would be shown by a failure to return to the original capacity. In one or two cases there seemed to be a very slight effect, but in all other cases it could not be detected. Since this 
TABLE VI

Lag of Capacity on Changing the Pressure

Condenser 4660R. April 7, 1908

\begin{tabular}{c|c|c}
\hline Pressure & Capacity & Time \\
\hline $760 \mathrm{~mm}$ & $0.49979 \mathrm{mf}$ & $11.30 \mathrm{a.m}$. \\
10 & 650 & 35 \\
10 & 607 & 40 \\
10 & 593 & 43 \\
10 & 583 & 46 \\
10 & 575 & 49 \\
10 & 566 & 52 \\
760 & 966 & 56 \\
760 & 972 & 59 \\
760 & 975 & 12.02 \\
\hline
\end{tabular}

is the case, a pressure coefficient can be computed which will apply to small changes of pressure. This has been done, and the results are given in Table VII. It is noticeable that in one case there is an increase of capacity with a decrease of pressure.

TABLE VII

Change of Capacity with Pressure

\begin{tabular}{c|c}
\hline Condenser & $\begin{array}{c}\text { Pressure coefficient of the capacity in parts per } \\
\text { hundred thousand per cm change in pressure }\end{array}$ \\
\hline 5471A 0.1 section & 2.0 \\
5 0.1 section & 2.0 \\
5471B 0.1 section & 1.7 \\
0.1 section & 1.0 \\
No. 1 & 0.5 \\
7765L & 2.0 \\
$7765 \mathrm{R}$ & -0.2 \\
$7766 \mathrm{~L}$ & 1.1 \\
$7766 \mathrm{R}$ & 3.4 \\
7763 & 0.3 \\
No. 12 & 1.5 \\
\hline
\end{tabular}

Measurements were made with 100 cycles A. C. $48848^{\circ}-10-3$ 
The cause of this change of capacity with change of pressure is very evident. At all times the pressure of the air is resisted by the elastic forces within the condenser. If the pressure is reduced, the condenser expands, thus increasing the distance between the plates and at the same time increasing the area of the plates. In most cases the increase in the distance between the plates produces the more marked effect, so that the capacity decreases with a decrease of pressure. However, the one condenser examined which is an exception to this rule, is very firmly clamped between heavy brass plates, so that changes in atmospheric pressure produce very little effect on the thickness of the condenser; but it can expand with decreasing pressure so as to increase the area of the plates and thus increase the capacity.

The magnitude of these effects is such that the daily fluctuations of the barometric pressure produces a change in the capacity which can be measured, provided all other influences are eliminated. To accomplish this, a bridge was set up in which both the auxiliary condenser and the standard capacity were kept in an exhausted desiccator. These and the condensers under test were kept in a chamber whose temperature was controlled by a thermostat. After one or two trials reasonably satisfactory results were obtained and are given in Table VIII. While the agreement between the observed values and those computed from the previously determined pressure coefficient is not all that could be desired, yet it is all that can reasonably be expected considering the difficulties involved.

\section{TABLE VIII}

The Change in Capacity of Certain Condensers from 10.30 a. m., February 11, to 9.20 a. m., February 12, 1910

The Barometric Pressure Changed from $766 \mathrm{~mm}$ to $744 \mathrm{~mm}$. The Temperature was Maintained Constant by a Thermostat

\begin{tabular}{c|c|c}
\hline Condenser & $\begin{array}{c}\text { Decrease in capacity in parts } \\
\text { per million }\end{array}$ & Computed decrease \\
\hline $7765 \mathrm{~L}$ & 10 & 18 \\
$7766 \mathrm{~L}$ & 44 & 24 \\
No. 6 & 2 & 12 \\
" 7 & 6 & 6 \\
“ 8 & 4 & 6 \\
\hline
\end{tabular}

All measurements were made at 100 cycles A. C. 
The above results show that, while the ordinary fluctuations of barometric pressure produce only very small effects, yet in case there is any considerable change in pressure, careful account must be taken of it in work of the highest precision.

The effect of changes of the atmospheric humidity upon the capacity was also investigated with negative results. A condenser was even placed in water and left there for forty-eight hours without producing a measurable effect upon the capacity.

\section{THE EFFECT OF VOLTAGE}

When compared with an air condenser, the capacity of some mica condensers depends upon the voltage used in making the comparison. In the case of mica condensers built up in the usual way with tin-foil sheets interleaved with sheets of mica, the effect is always small; and, in the case of the better condensers, can not be detected. However, with silvered mica condensers the effect is very pronounced in every case which has been examined. Moreover there is a certain instability, in that with the same voltage different capacities will be obtained at different times. This effect is illustrated in the curve of Fig. I3, where the total increase in capacity on increasing the voltage from 20 to Ioo volts is approximately o.I per cent.

While a condenser of this type can not be considered in selec ing a standard, yet it seemed desirable to determine the cause of such a marked effect. On examining some sheets of silvered mica it was observed that there were flakes on the surface underneath which the plating solution only partially penetrated. On removing these and examining the surface under a microscope it was found that there were places underneath these flakes where there seemed to be spots of silver which were imperfectly connected with the main coating of silver. A chemical test showed that these spots contained silver.

In view of these facts the following explanation seems plausible. As the spots of silver are imperfectly connected to the plates of the condenser, at low voltage they will play no part in the charging of the condenser. As the voltage is increased there will be more and more cases where the charge will cross the intervening space, thus charging the spot of silver. In this way the virtual thickness of the plate is decreased, hence the capacity is increased. 
If this explanation is correct, it would seem probable that at very high frequencies there would be little tendency to charge the small spots of silver at any voltage, so that there would be little change with voltage at high frequency. However, if the charging and discharging takes place slowly, the potential of the

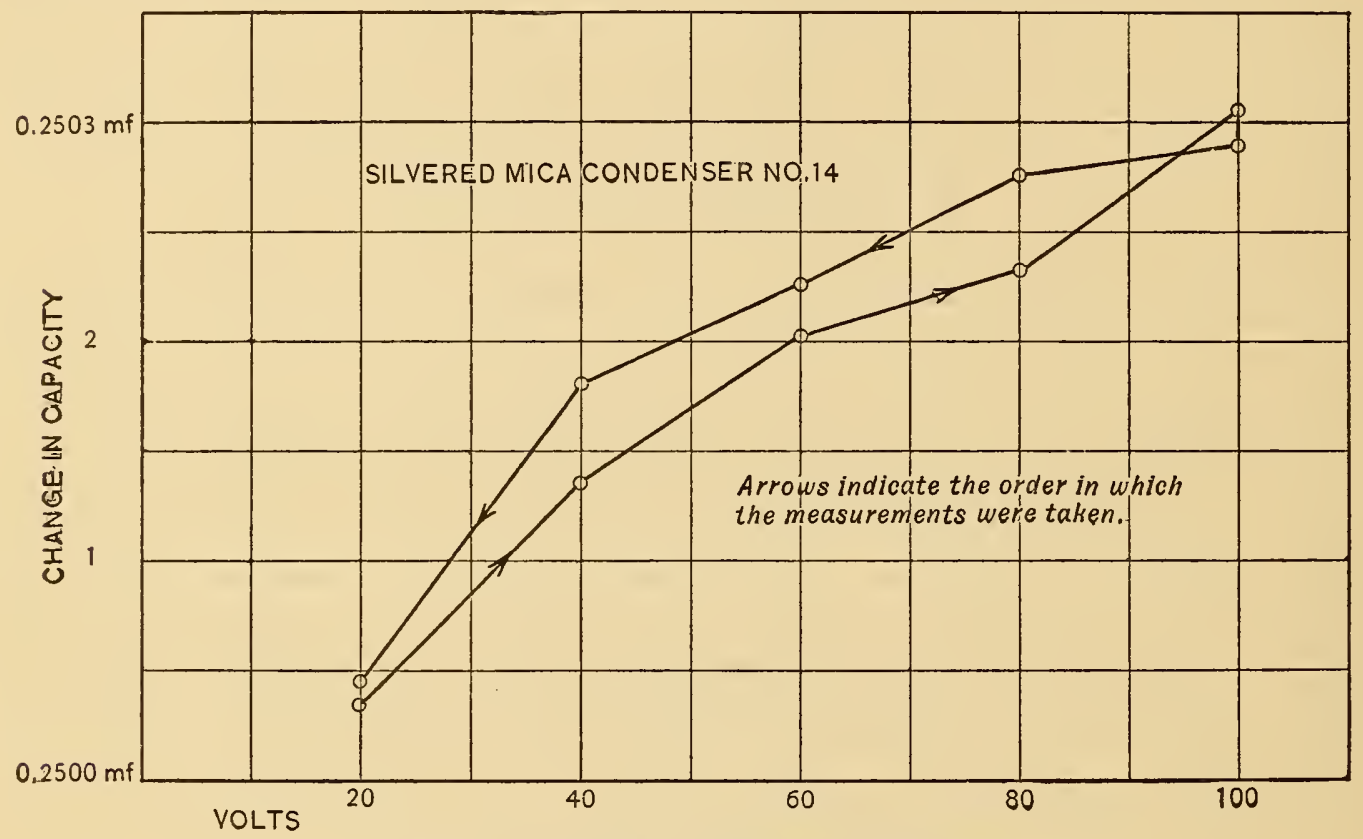

Fig. 13.-Change in capacity with voltage.

spots will be but little different from that of the plates at any time during the cycle. In this case also there would be little change over a moderate range of voltage. Thus we would expect a maximum effect at some intermediate frequency. That this is the case is indicated by Table IX.

TABLE IX

Effect of Frequency on the Increase in Capacity of Silvered Mica Condensers with Increasing Voltage

\begin{tabular}{l|c|c|c}
\hline \multirow{2}{*}{$\begin{array}{c}\text { Time of discharge in } \\
\text { D. C. }\end{array}$} & Frequency with A. C. & $\begin{array}{c}\text { Increase in capacity on increasing the voltage from } 30 \\
\text { to } 70 \text { volts, in parts per hundred thousand }\end{array}$ \\
\cline { 2 - 3 } & & Condenser No. 13 & Condenser No. 14 \\
\hline & 3000 & 16 & 40 \\
1000 & 26 & 76 \\
100 & 50 & 50 \\
& 50 & 36 & 36 \\
0.1 & 18 & 10 \\
0.5 & 12 & \\
\hline
\end{tabular}


One would also expect that the change in capacity with changing frequency would be more pronounced with such a condenser than with ordinary condensers. However, this is so small in comparison with the change in capacity due to absorption, that it can not be determined whether or not any of the observed change is due to this phenomenon.

\section{THE STABILITY OF MICA CONDENSERS}

It has been shown by Rosa and Grover that unclamped condensers are very unstable. It seemed desirable to see to what extent clamping a condenser improves it in this respect. Accordingly a number of condensers were twice carried through the temperature cycle from $15^{\circ}$ to $30^{\circ} \mathrm{C}$. The curves of Fig. I4 are fairly representative of the results. It will be seen that there is an uncertainty which often amounts to several parts in ten thousand. In some cases there is an increase in capacity on returning to $15^{\circ} \mathrm{C}$, in others a decrease. While this is a great improvement over unclamped condensers, yet it was evident that mica condensers must be maintained at constant temperature if they are to be used in work of precision. It seemed quite possible also that condensers which are shielded from changes in the barometric pressure will be more constant than those which are not. To test this point four condensers were sealed in a desiccator and the air exhausted. In the first attempts the ground joint began to leak in three or four weeks, and three months elapsed before a satisfactory method of sealing the desiccator was devised. However, with the aid of Dr. Nutting, the desiccator was finally sealed in such a manner that for more than a year there has been so little change in pressure that it can not be detected by the Plücker tube which is attached to it. The total pressure is approximately a millimeter. These condensers with others have been constantly maintained at a temperature of $25^{\circ} \mathrm{C}$.

One of these condensers (No. I2) is very sensitive at low pressures to any change in pressure, though at atmospheric pressure the pressure coefficient is not unusually large ( 1.5 parts per hundred thousand per centimeter change in pressure). In the first attempts at keeping the condensers in a vacuum, I was always able to detect the appearance of a leak by the change in capacity of this condenser. This sensitiveness to ehanges in pressure is fur- 


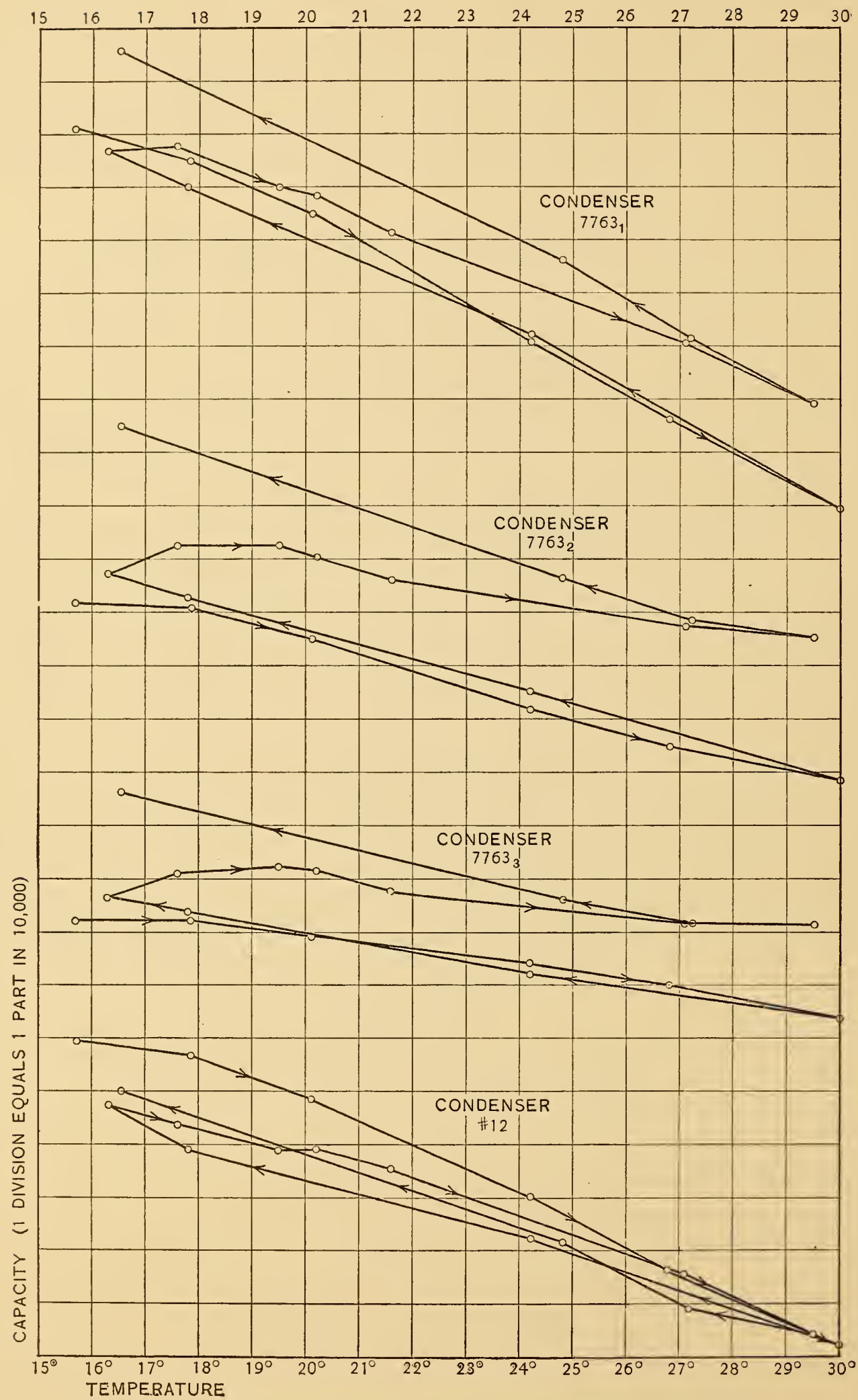

Fig. 14.-Curves showing the instability of clamped condensers 
ther indicated by the fact that the decrease in capacity of No. I 2 on going from atmospheric pressure to a vacuum is nearly 3 per cent, while for the other condensers it is less than o.I per cent. The plates between which the condenser is clamped are much thinner than are those of the other condensers, though it is not known that this is the cause for the exceptional behavior. For this reason the values of No. I 2 are given much less weight than those of the other condensers in the vacuum desiccator.

In order to determine the behavior of condensers under different methods of treatment, a series of measurements extending over nearly five months was made upon the following condensers: Three condensers in a vacuum, maintained at approximately $25^{\circ} \mathrm{C}$; five condensers, known by previous tests to be very stable, also maintained at $25^{\circ} \mathrm{C}$ under atmospheric pressure; four good condensers subjected to the ordinary variations of room temperature. After a few measurements it was evident that the condensers in the vacuum were relatively more constant than any of the others. Hence the mean of these three is taken as remaining constant, and all other values are referred to them. These condensers were put in a vacuum on March 5, r9o9. The first measurements were made on March 9, but they did not seem to have adjusted themselves to the change in pressure until March $\mathrm{I}_{3}$. The temperature coefficients of the condensers which were left at room temperature had been previously determined, so that the temperature and capacity were measured, and the capacity at $20^{\circ} \mathrm{C}$ was computed from these measurements.

The results of these measurements are shown in the curves of Fig. I5. Those condensers which are in a vacuum are somewhat more stable than any of the others, though the improvement over those which are kept at constant temperature is not marked. However, both of these show a decided improvement over those which are kept at room temperature.

It should be stated that an accuracy of measurement of one part in a hundred thousand was in all cases attained, and often somewhat exceeded. The changes which appear are greater than the experimental errors. These changes may be due to errors in the temperature, for a condenser is a poor conductor of heat and the temperature is measured outside the condenser proper. Even with those controlled by a thermostat there is always a possi- 


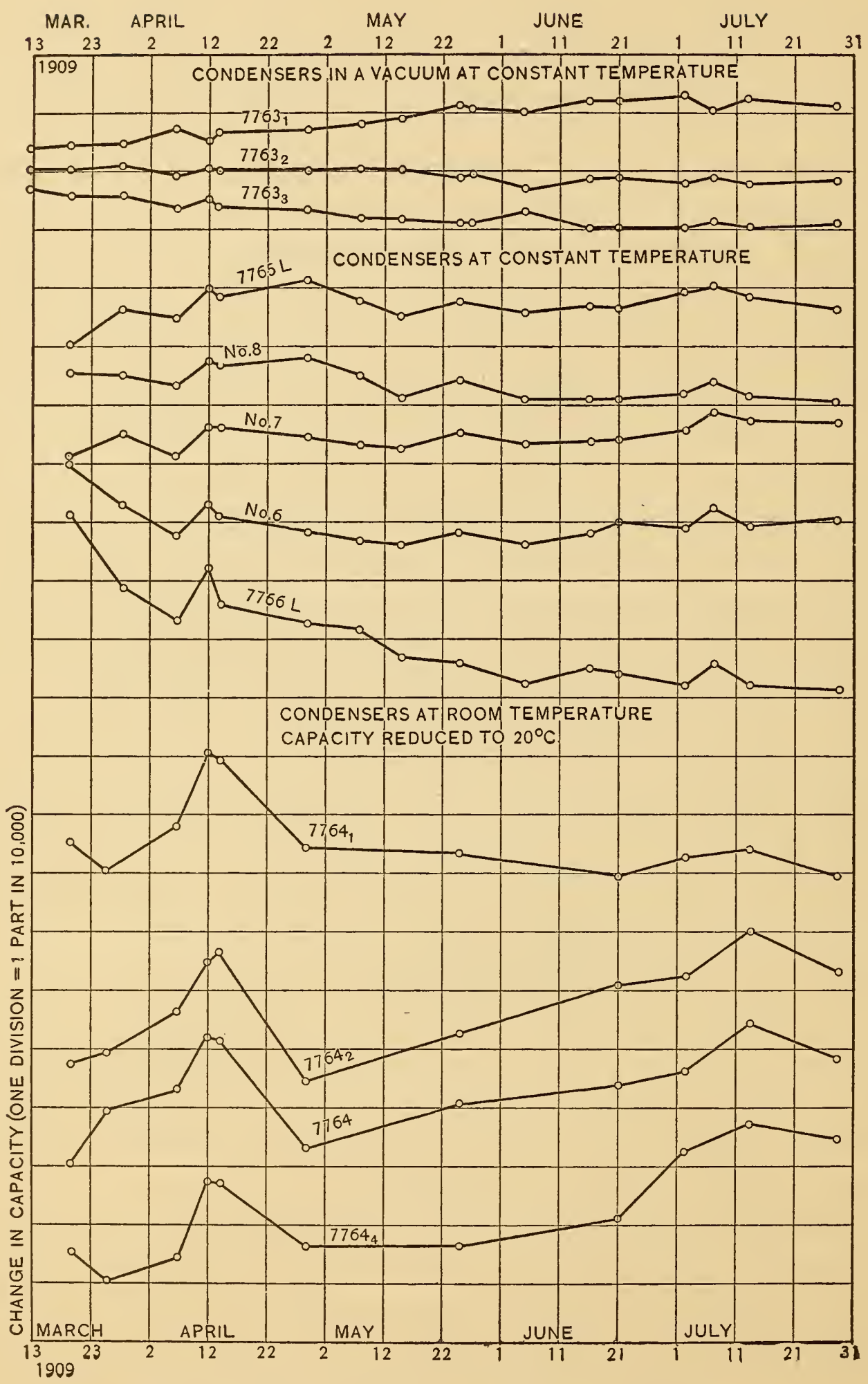

Fig. 15.-Curves showing the change of capacity with time 
bility that slight temperature variations have occurred. On the other hand it should not be overlooked that these changes may be due to some cause of which we have no knowledge.

With the best condensers maintained at a constant temperature a constancy of one part in ten thousand over several months was attained. With those in a vacuum this was somewhat improved upon, so that the fluctuations are only a few parts in a hundred thousand, and these have taken place slowly. It should be noticed that one of the condensers, 7766L, which was maintained at constant temperature, showed a slow but continuous decrease in capacity. The condensers which were kept at room temperature showed fluctuations of two or three parts in ten thousand.

It should be noticed that the condensers in a vacuum are the same condensers as those whose behavior is shown in the previous curves (Fig. I4), where considerable variations in a comparatively short time are observed. It would seem to be established by these measurements, and it has been corroborated by several other series, that a condenser at room temperature shows fluctuations in its capacity amounting to several parts in ten thousand. However, the best condensers, when kept at constant temperature, will ordinarily remain constant to a part in ten thousand for a considerable time, and they will show even greater constancy if they are sealed in a vacuum.

\section{THE RELATIONSHIP BETWEEN THE DIFFERENT APPARENT CAPACITIES}

The principal cause for the difference in the apparent capacities of a condenser is, without doubt, its absorption. At the very high frequencies used in wireless telegraphy the question of the distribution of the charge on the condenser plates becomes a matter for consideration; but, as will be shown later, below 3000 cycles it need not be considered. Also in the case of silvered-mica condensers, and doubtless some others, the voltage may affect the apparent capacity for reasons already explained. But for the present no condenser in which this defect is known to exist will be considered. Hence the problem is to see whether the known facts in regard to absorption can explain the observed differences in capacity. 


\section{QUALITATIVE RELATIONS}

Before attempting to reconcile all the differences in a rigid manner, some qualitative deductions from the curves of Figs. 7-I 2 may be of use. One of the most important conclusions that will be evident from an examination of these curves is that it can not be readily predicted from A. C. measurements at ordinary frequencies what a condenser will do when measured by D. C. with relatively long times of charge and discharge. There are condensers whose capacity changes but little with change of frequency on A. C., yet which do show a considerable change with length of discharge; though in the majority of cases if a condenser shows little change on A. C. it shows little change on D. C., and if it shows a relatively large change on $\mathrm{A}$. $\mathrm{C}$. it also shows a large change on D. C. Hence there seems to be no simple way of connecting the changes on $\mathrm{A}$. C. with those on $\mathrm{D}$. C.

However, the phase difference always gives an indication of the magnitude of the change in the A. C. capacity with change of frequency. One can not express this relationship accurately, but in all cases where the phase difference is large, there is a considerable change of capacity with change of frequency. Also the behavior in D. C. measurements may be roughly predicted from the value of the absorption. When a condenser has a large measured absorption, then its increase of capacity with increasing time of discharge is also large. Hence a knowledge of both the phase difference and the absorption of a condenser at any temperature, will give a fair idea of the behavior of the condenser at that temperature. The question then arises whether two or more constants can be found by means of which all the facts in regard to a condenser can be expressed.

\section{APPLICATION OF MAXWELL'S THEORY OF ABSORPTION TO DIRECT- CURRENT MEASUREMENTS}

Since the change in capacity is due to absorption, it is desirable to ascertain whether the necessary constants can not be deduced from one of the theories which have been proposed to explain this phenomenon. Several theories have been advanced, but the only undisputed one was proposed by Maxwell. ${ }^{12}$ He showed that with

${ }^{12}$ Maxwell, Electricity and Magnetism, $\S 328$. 
a nonhomogeneous dielectric in which the product of the dielectric constant and the specific resistance are different for the different parts of the dielectric, there will be absorption. The question is whether this is of a magnitude sufficient to account for the observed effect. In order to discuss this more satisfactorily, a brief discussion of Maxwell's reasoning will be given.

NOMENCLATURE.

$a_{1}$ and $a_{2}$ are thicknesses.

$K_{1}$ and $K_{2}$ are the dielectric constants. $\rho_{1}$ and $\rho_{2}$ are the specific resistances.

$E_{1}$ and $E_{2}$ are the instantaneous differences of potential.

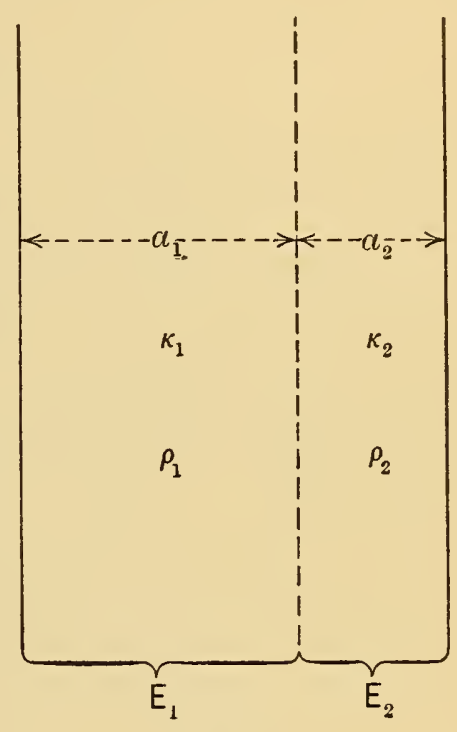

Fig. 16.

Consider for simplicity a condenser composed of two dielectrics in parallel strata, each of which has I sq. cm surface. It is easily shown that it is immaterial whether there are only two strata, one of each dielectric, or a number of strata of each material, whose combined thickness is the same as the single stratum. Therefore the case considered will cover the case of two dielectrics, provided that they lie in strata. The equations are easily modified in case there are more than two dielectrics, and the final conclusions apply to any number of dielectrics.

Let an emf, $E$, be applied to the terminals. Then a quantity $Q$ will be instantaneously displaced through the dielectric, producing potential differences of $E_{1}$ and $E_{2}$ between the faces of the respective strata. If we consider only the left-hand stratum and designate the capacity due to it by $C_{1}$, then

$$
Q=C_{1} E_{1}=\frac{E_{1} K_{1}}{4 \pi a_{1}}
$$




$$
\begin{aligned}
& \text { Also } Q=C_{2} E_{2}=\frac{E_{2} K_{2}}{4 \pi a_{2}} \\
& \text { Hence } \frac{Q}{C}=E=E_{1}+E_{2}=Q\left\{\frac{4 \pi a_{1}}{K_{1}}+\frac{4 \pi a_{2}}{K_{2}}\right\}
\end{aligned}
$$

where $C$ is the capacity of the condenser due to an instantaneous charge.

$$
\therefore \frac{\mathrm{I}}{\mathrm{C}}=4 \pi\left(\frac{a_{1}}{K_{1}}+\frac{a_{2}}{K_{2}}\right)
$$

If the emf is applied for a finite time, it is necessary to consider the effect of conduction through the dielectric. In this case $E_{1}$ and $E_{2}$ are not constants, but vary with the time, though at all times $E_{1}+E_{2}=E$. If $i$ represents the total current through the dielectric at any instant, $i_{1}{ }_{1}$ the conduction current through the left-hand dielectric, $i^{\prime \prime}{ }_{1}$ the displacement current, and $\rho_{1}$ the specific resistance,

$$
\begin{aligned}
i^{\prime}{ }_{1} & =\frac{E_{1}}{a_{1} \rho_{1}} \\
\text { and } i^{\prime \prime}{ }_{1} & =\frac{d Q}{d t}=\frac{K_{1}}{4 \pi a_{1}} \frac{d E_{1}}{d t} \\
\therefore \quad i & =i^{\prime}{ }_{1}+i^{\prime \prime}{ }_{1}=\frac{E_{1}}{a_{1} \rho_{1}}+\frac{K_{1}}{4 \pi a_{1}} \frac{d E_{1}}{d t}
\end{aligned}
$$

In a like manner$$
i=i_{2}^{\prime}+i^{\prime \prime}{ }_{2}=\frac{E_{2}}{a_{2} \rho_{2}}+\frac{K_{2}}{4 \pi a_{2}} \frac{d E_{2}}{d t}
$$

and

$$
\frac{E_{1}}{a_{1} \rho_{1}}+\frac{K_{1}}{4 \pi a_{1}} \frac{d E_{1}}{d t}=\frac{E_{2}}{a_{2} \rho_{2}}+\frac{K_{2}}{4 \pi a_{2}} \frac{d E_{2}}{d t}
$$

since the total current in each dielectric is the same. If, now, the emf, $E$, is maintained until an equilibrium is reached,

$$
\frac{d E_{1}}{d t}=\mathrm{o} \text { and } \frac{d E_{2}}{d t}=\mathrm{o} .
$$

Let $E_{\infty} E_{1}$ and ${ }_{\infty} E_{2}$ represent the values of $E_{1}$ and $E_{2}$ when the equilibrium condition is reached. Then

$$
i=\frac{{ }_{\infty} E_{1}}{a_{1} \rho_{1}}=\frac{{ }_{\infty} E_{2}}{a_{2} \rho_{2}}=\frac{E_{1} E_{1}+{ }_{\infty} E_{2}}{a_{1} \rho_{1}+a_{2} \rho_{2}}=\frac{E}{R}
$$

where $R$ is the total resistance between the plates of the condenser. 
Let us suppose that after this condition has been reached, the outside emf, $E$, is removed, the terminals of the condenser connected together by a conductor of negligible resistance for a time just sufficient to allow the free charge $Q$ to neutralize, and then both terminals insulated. Let ${ }_{0} E_{1}$ and ${ }_{0} E_{2}$ be the values of $E_{1}$ and $E_{2}$ at the instant the terminals are insulated.

Then ${ }_{0} E_{1}+{ }_{0} E_{2}=0$

And

$$
\begin{aligned}
& { }_{0} E_{1}={ }_{\infty} E_{1}-\frac{4 \pi a_{1}}{K_{1}} Q \\
& \left.{ }_{0} E_{2}={ }_{\infty} E_{2}-\frac{4 \pi a_{2}}{K_{2}} Q\right\} \\
& { }_{\infty} E_{1}+{ }_{\infty} E_{2}=E=4 \pi Q\left(\frac{a_{1}}{K_{1}}+\frac{a_{2}}{K_{2}}\right)
\end{aligned}
$$

A comparison of this with equation (I I) shows that the instantaneous discharge is the same as the instantaneous charge. If $C$ represents the geometric capacity,

$$
C=\frac{E}{Q}=4 \pi\left(\frac{a_{1}}{K_{1}}+\frac{a_{2}}{K_{2}}\right)
$$

It may be measured by either the instantaneous charge or the instantaneous discharge.

After the terminals are insulated the current $i$ is zero, hence from equation (I 5 ),

$$
-\frac{d E_{1}}{E_{1}}=\frac{4 \pi}{\rho_{1} K_{1}} d t
$$

Integrating, $\log E_{1}=-\frac{4 \pi t}{\rho_{1} K_{1}}+a$ constant.

Since ${ }_{0} E_{1}$ is the value of $E_{1}$ when $t=0$

$$
E_{1}={ }_{0} E_{1} e^{-\frac{4 \pi t}{\rho_{1} K_{1}}}
$$


Likewise $E_{2}={ }_{0} E_{2} e^{-\frac{4 \pi t}{\rho_{2} K_{2}}}$

The difference of potential $E^{\prime}$ between the terminals at any instant, $t$, is

$$
E^{\prime}=E_{1}+E_{2}={ }_{0} E_{1} e^{-\frac{{ }_{4} 4 \pi t}{\rho_{1} K_{1}}}+{ }_{0} E_{2} e^{-\frac{4 \pi t}{\rho_{2} K^{2}}}
$$

The values of ${ }_{0} E_{1}$ and ${ }_{0} E_{2}$ are given in equations (19). From (18)

$$
\begin{gathered}
{ }_{\infty} E_{1}=\frac{E a_{1} \rho_{1}}{R} \text { and }{ }_{\infty} E_{2}=\frac{E a_{2} \rho_{2}}{R} \\
\therefore \quad E^{\prime}=E\left\{\left(\frac{a_{1} \rho_{1}}{R}-\frac{4 \pi a_{1} C}{K_{1}}\right) e^{-\frac{4 \pi t}{\rho_{1} K_{1}}}+\left(\frac{a_{2} \rho_{2}}{R}-\frac{4 \pi a_{2} C}{K_{2}}\right) e^{-\frac{4 \pi t}{\rho_{2} K^{2}}}\right\}
\end{gathered}
$$

This equation gives the value of the potential difference of the condenser plates at any time, $t$, after they are insulated in terms of the dielectric constants and specific resistances of the strata. For simplicity let

$$
\begin{array}{cc}
D_{1}=\frac{a_{1} \rho_{1}}{R}-\frac{4 \pi a_{1} C}{K_{1}} ; & D_{2}=\frac{a_{2} \rho_{2}}{R}-\frac{4 \pi a_{2} C}{K_{2}} \\
m_{1}=\frac{4 \pi}{K_{1} \rho_{1}} ; & m_{2}=\frac{4 \pi}{K_{2} \rho_{2}}
\end{array}
$$

Then $E^{\prime}=E\left\{D_{1} e^{-m_{1} t}+D_{2} e^{-m_{2} t}\right\}$

It should be noticed that $m_{1}$ and $m_{2}$ are necessarily positive, and it follows from (24) and (25) that $D_{1}+D_{2}=O$, since ${ }_{0} \mathrm{E}_{1}+{ }_{0} \mathrm{E}_{2}=0$.

The residual charge $A_{t}$ is defined as the ratio of the quantity which reappears when a condenser has been completely charged, then instantly discharged, and after this left insulated for a time, $t$; to the total free charge.

Hence, from the equation (26),

$$
A_{t}=\frac{E^{\prime} C}{E C}=D_{1} e^{-m_{1} t}+D_{2} e^{-m_{2} t}
$$

This can be put into a somewhat simpler form for purposes of computation by expanding in a series. Then

$$
\begin{aligned}
A_{t} & =D_{1}\left(\mathrm{I}-m_{1} t+\frac{m^{2}{ }_{1} t^{2}}{2 !}-\frac{m^{3}{ }_{1} t^{3}}{3 !}+\ldots \ldots\right) \\
& +D_{2}\left(\mathrm{I}-m_{2} t+\frac{m^{2}{ }_{2} t^{2}}{2 !}-\frac{m^{3}{ }_{2} t^{3}}{3 !}+\ldots \ldots\right)
\end{aligned}
$$


Now, $D_{1}+D_{2}=0$

$$
\text { And }-\log \left(\mathrm{I}+m_{1} t\right)=-m_{1} t+\frac{m^{2}{ }_{1} t^{2}}{2}-\frac{m^{3}{ }_{1} t^{3}}{3}+\ldots
$$

Hence, if $m_{1} t$ and $m_{2} t$ are sufficiently small so that terms above the square may be neglected,

$$
A_{t}=-D_{1} \log \left(\mathrm{I}+m_{1} t\right)-D_{2} \log \left(\mathrm{I}+m_{2} t\right)
$$

This should hold with considerable accuracy during the first part of the discharge, but can not be used when $t$ is relatively large.

We shall now apply a numerical test to this formula, to see whether it explains the observed phenomena. In the adjustment of some mica condensers, the capacity of a single sheet of mica was determined in a number of cases. These measurements gave a mean value in electrostatic units of about $105 \mathrm{~cm}$ per sq. $\mathrm{cm}$. Also from measurements of the insulation resistance, the mean resistance, $R$, per sq. cm was found to be $8.3 \times \mathrm{IO}^{2}$ electrostatic units. The thickness of the mica sheets being about $0.005 \mathrm{~cm}$, and the known dielectric constant of paraffin about 2, and of mica 7 , the thickness of the paraffin is computed to be $0.000 \mathrm{~cm}$. An examination of the plates shows that this is a reasonable value. Since, now, the insulation resistance of paraffin is very high, being usually given as between $\mathrm{IO}^{18}$ and $\mathrm{IO}^{19}$ ohms per $\mathrm{cm}$ cube or $1 \mathrm{I}^{6}$ to ${ }^{1} \mathrm{O}^{7}$ electrostatic units, it follows that a very large part of the observed insulation resistance is due to the paraffin, so that the resistance of the mica can not be computed from this data, since there may be a wide variation in the resistance of the mica without producing any appreciable difference in the total insulation resistance. The published values of the specific resistance of mica vary from $\mathrm{IO}^{-1}$ to $\mathrm{IO}^{4}$ e!ectrostatic units. The lower values, which are due to Rood ${ }^{13}$ should not be given equal weight with the others, as he did not continue the application of the emf until a steady state was reached. Values obtained by Grover in this laboratory are in accord with those of Curie ${ }^{14}$ who found ${ }^{4} \mathrm{O}^{4}$ electrostatic units. The value of the residual charge $A_{0 \cdot 1}$ at the end of a tenth of a second, computed from the data already given and using various values of the specific resistance of mica are given in Table $\mathrm{X}$.

${ }^{13}$ Amer. J. Sci. 164, p. I6 I; $1902 . \quad{ }^{14}$ Annal. Chim. Phys. (6), 18, p. 229; 1889. 


\section{TABLE X}

Given a Condenser with Paraffin and Mica as Dielectrics, to Compute the Residual Charge at the end of a Tenth of a Second, Using the Formula of Equation (30)

CONSTANTS: $\mathrm{R}=10^{3}$ ELECTROSTATIC UNITS; $\mathrm{C}=105 \mathrm{CM}$

\begin{tabular}{|c|c|c|c|c|}
\hline \multicolumn{4}{|c|}{ Mica } & Paraffin \\
\hline \multicolumn{4}{|c|}{$\begin{aligned} a_{1} & =0.005 \mathrm{~cm} \\
K_{1} & =7\end{aligned}$} & \multirow{8}{*}{$\begin{aligned} a_{2} & =0.0001 \mathrm{~cm} \\
K_{2} & =2 \\
\rho_{2} & =10^{7} \text { electrostatic units } \\
m_{2} t & =6.4 \times 10^{-8} \\
D_{2} & \log \left(1+m_{2} t\right) \text { is negligible }\end{aligned}$} \\
\hline$\rho_{1}$ & $\mathrm{~m}_{1} \mathrm{t}$ & $\mathrm{D}_{1}$ & $\mathbf{A}_{0.1}$ & \\
\hline $10^{4}$ & $1.8 \times 10^{-5}$ & -0.90 & 0.000016 & \\
\hline $10^{3}$ & $1.8 \times 10^{-4}$ & -0.94 & 0.00017 & \\
\hline $10^{2}$ & $1.8 \times 10^{-3}$ & -0.95 & 0.0017 & \\
\hline 50 & $3.6 \times 10^{-3}$ & -0.95 & 0.0034 & \\
\hline 15 & $1.2 \times 10^{-2}$ & -0.95 & 0.005 & \\
\hline 10 & $1.8 \times 10^{-2}$ & -0.95 & 0.017 & \\
\hline
\end{tabular}

From the curves in Figs. 7-I I an idea of the value of the absorbed charge which is actually given up during the first tenth of a second can be obtained in the following way. As will be shown later, the value of the capacity at 1200 cycles differs but little from the geometric capacity. The difference between the apparent capacity using 0.6 second charge and o.I second discharge and the geometric capacity is due to the absorbed charge which reappears in the first o.I second. The value of this varies from 0.3 to I per cent in the case of these condensers. Hence, if the computed values are to agree with the observed, the specific resistance of mica must lie between ro and 50 electrostatic units. As the best experimental results give in the neighborhood of $\mathrm{IO}^{4}$ electrostatic units, it seems probable that only a small part of the observed absorption is due to this cause. 
12. APPLICATION OF MAXWELL'S THEORY OF ABSORPTION TO ALTERNATING-CURRENT MEASUREMENTS

A confirmation of the conclusions drawn from the D. C. measurements can be obtained by applying the theory to A. C. measurements. The formulas were first derived by Rowland, ${ }^{15}$ though he did not apply them to actual measurements. In the following derivation the nomenclature of Fig. 16 will be followed as far as possible:

From equation ( 17 )

$$
i=\frac{E_{1}}{a_{1} \rho_{1}}+\frac{K_{1}}{4 \pi a_{1}} \frac{d E_{1}}{d t}=\frac{E_{2}}{a_{2} \rho_{2}}+\frac{K_{2} d E_{2}}{4 \pi a_{2} d t}
$$

Let

$$
m_{1}=\frac{4 \pi}{K_{1} \rho_{1}} \text { and } B_{1}=\frac{4 \pi a_{1}}{K_{1}}
$$

with similar values for $m_{2}$ and $B_{2}$,

then

$$
\frac{d E_{1}}{d t}+m_{1} E_{1}=B_{1} i
$$

If the current follows the sine law,

$$
\begin{aligned}
& i=I \sin p t \text { where } p=2 \pi \times \text { the frequency } \\
& \therefore \frac{d E_{1}}{d t}+m_{1} E_{1}=B_{1} I \sin p t
\end{aligned}
$$

Integrating $E_{1}=\frac{I B_{1}}{m_{1}{ }^{2}+p^{2}}\left(m_{1} \sin p t-p \cos p t\right)+A e^{-m_{1} t}$

where $A$ is the constant of integration. The last term becomes zero when $t$ is sufficiently large. In the same manner

$$
E_{2}=\frac{I B_{2}}{m_{2}^{2}+p^{2}}\left(m_{2} \sin p t-p \cos p t\right)
$$

But the emf also follows the sine law, so that

$$
E_{1}+E_{2}=E \sin (p t-\phi)
$$

where $\phi$ is the difference in phase of the current and emf. Substituting the values of $E_{1}$ and $E_{2}$ from (34) and (35) 


$$
\begin{gathered}
E \sin (p t-\phi)=I\left\{\frac{B_{1}}{m_{1}^{2}+p^{2}}\left(m_{1} \sin p t-p \cos p t\right)\right. \\
\left.+\frac{B_{2}}{m_{2}^{2}+p^{2}}\left(m_{2} \sin p t-p \cos p t\right)\right\}
\end{gathered}
$$

Since this is true for all values of $p t$, it is true when $p t=o$ and when $p t=\frac{\pi}{2}$

In the first case

$$
E \sin \phi=I p\left\{\frac{B_{1}}{m_{1}^{2}+p_{2}}+\frac{B_{2}}{m_{2}^{2}+p^{2}}\right\}
$$

In the second case

$$
E \cos \phi=I\left\{\frac{m_{1} B_{1}}{m_{1}^{2}+p^{2}}+\frac{m_{2} B_{2}}{m_{2}^{2}+p^{2}}\right\}
$$

From Fig. 3, page 437 , it is seen that

$$
\frac{\mathrm{I}}{p C}=\frac{E \sin \phi}{I}
$$

where $C$ is the apparent capacity. Hence, from equation (37)

$$
\frac{\mathrm{I}}{C}=\frac{B_{1}}{\mathrm{I}+\frac{m_{1}{ }^{2}}{p^{2}}}+\frac{B_{2}}{\mathrm{I}+\frac{m_{2}{ }^{2}}{p^{2}}}
$$

If the frequency is infinite

$$
\stackrel{\mathrm{I}}{C}=B_{1}+B_{2}=4 \pi\left(\frac{a_{1}}{K_{1}}+\frac{a_{2}}{K_{2}}\right)
$$

which is the same value as found for instantaneous charge or discharge.

In equations (37) and (38), $p^{2}+m_{1}{ }^{2}$ and $p^{2}+m_{2}{ }^{2}$ enter in the denominator, where

$$
m_{1}=\frac{4 \pi}{K_{1} \rho_{1}} \text { and } m_{2}=\frac{4 \pi}{K_{2} \rho_{2}}
$$

In all cases $K$ is greater than unity, and in the cases we are considering $\rho$ is still larger. Hence, if $p$ is more than Ioo, in no case will an error greater than I per cent be introduced by 
neglecting $\mathrm{m}^{2}$. Making this approximation and dividing (38) by $(37)$

$$
\cot \phi=\tan \theta=\frac{m_{1} B_{1}+m_{2} B_{2}}{p\left(B_{1}+B_{2}\right)}
$$

Hence, with the values of $K$ and $\rho$ which have been assumed, it follows from this theory that the phase difference should vary inversely as the frequency. This is equivalent to saying that the energy loss per second is independent of the frequency. The values of the phase difference given in the curves of Figs. 7-1 2 show very plainly that this is not the case.

Interesting results are also obtained by inserting the constants which make the computed value of the residual charge agree with the observed value in the formulas which have just been developed. These results are given in Table XI. It will be seen that the computed change in the capacity is very much less than the observed. On the other hand, the computed values of the phase difference at

\section{TABLE XI}

The Observed and Computed Values of the Phase Difference and the Change in Capacity at Different Frequencies

Formulas (39) and (41) are Used in the Computation

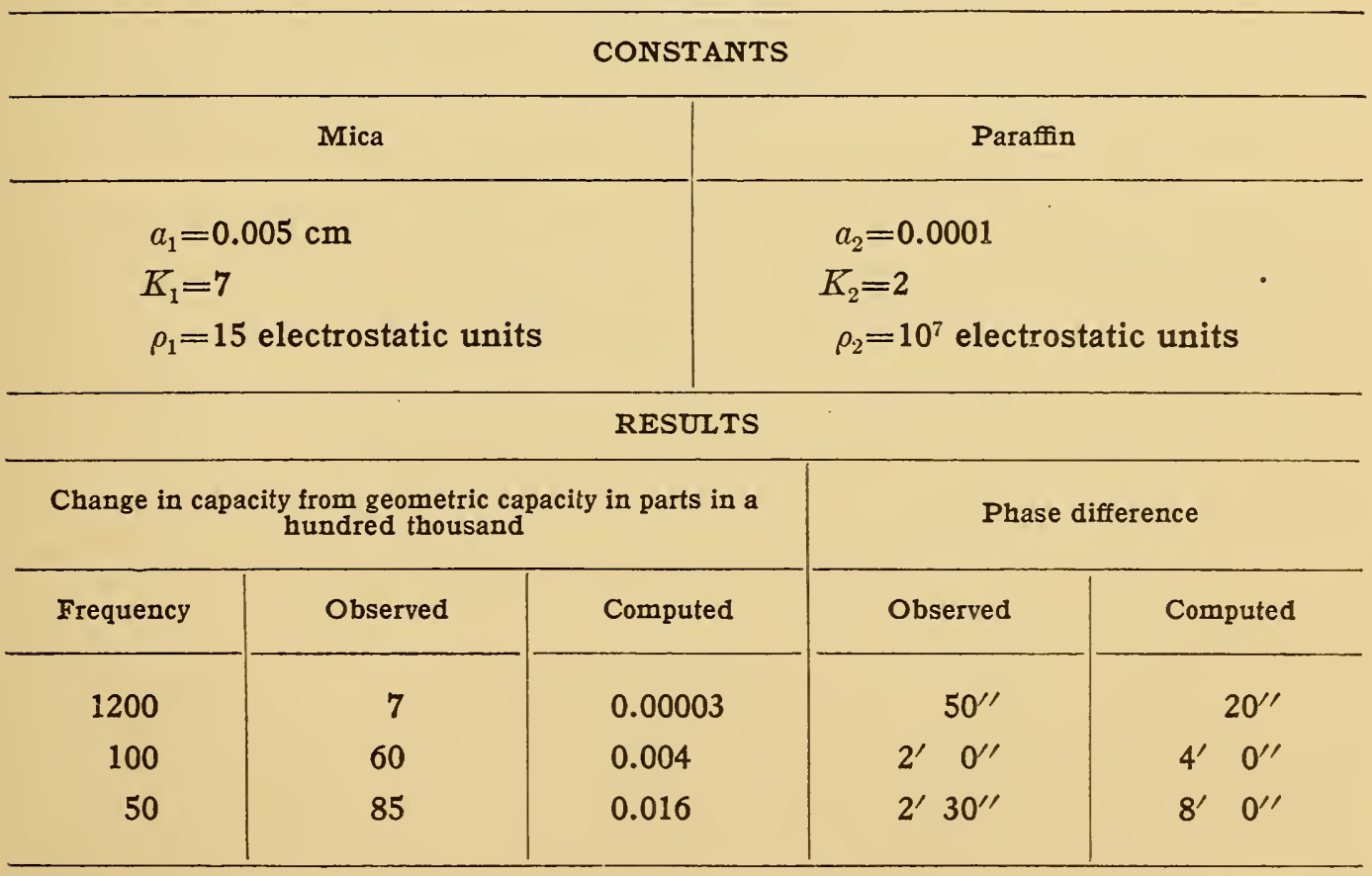

The observed values given are those of $5471 \mathrm{~B} \Sigma 0.1$. 
low frequencies is larger than that observed. This would indicate that the value of the specific resistance of mica has been taken too low, which is what the direct measurements of the resistance indicated.

From the above considerations it is evident that the observed absorption in mica condensers can not be accounted for on the basis of a stratified dielectric. If the true values of the constants are those which at present seem to be the most probable values, the absorption caused by the nonhomogeneity of the dielectric is a negligible part of the total absorption.

There remains the possibility suggested by Rowland ${ }^{16}$ that this theory be modified by supposing that electrolysis and thermoelectric currents are present. Still another theory ${ }^{17}$ has been advanced, which accounts for the phenomena of absorption by supposing that there is a polarization in the dielectric. Neither of these theories is sufficiently developed from a mathematical view point to allow of a numerical test. Hence the data which have been collected on the dependence of the apparent capacity on the method of measurement will be discussed from an empirical standpoint alone.

\section{DETERIMINATION OF THE GEOMETRIC CAPACITY}

If the various values of the capacity are to be connected by some relation, it is necessary first of all to know whether the geometric capacity as determined by A. C. is the same as that determined by D. C. The A. C. value is found by plotting values of the apparent capacity as ordinates and values of the period (reciprocal of the frequency) as abscissa. This curve is projected backward to intersect the axis of zero period, and the intercept on this axis is taken as the geometric capacity. In the case of the better condensers there is no difficulty in determining the geometric capacity with precision.

Examples are given in the curves of Fig. 17 . Here the frequencies used are 1200,100 , and 50 . Frequencies from 10 to 3000 were available, but neither the highest nor lowest were found to be useful, for reasons which follow. Above 900 cycles the only indicating instrument at hand was a telephone. Above rooo vibrations per second the sensibility of the ear decreases rapidly, so that

${ }^{16}$ Amer. J. of Math., 1, 53; 1878.

${ }^{17}$ First suggested by Kohlrausch, Pogg. Am., 91, p. 56; 1854 . 
Curtis.] Mica Condensers as Standards of Capacity.



Fig. 17.-Curves showing method of determining the geometric capacity by A.C. 
it is impossible to make measurements of the highest accuracy at frequencies very much above this. Also at 3000 cycles the inductance of shortest possible leads often introduces an appreciable reactance, for which a correction must be introduced; and the resistance of the leads may prevent the condenser from becoming completely charged during each cycle, necessitating still another correction. The measurements made at 3000 cycles, using all possible precautions, showed a small decrease in the apparent capacity over that measured at I 200 cycles, and there is nothing to indicate that the capacity so determined does not lie on the curve as drawn. However, on account of the inferior accuracy, the use of these values is more of a hindrance than a help in determining the geometric capacity.

Also a number of condensers were measured at 12.5 cycles. The results in this case are interesting and are shown in the curves of Fig. I 8. However, at this frequency the measurements are not very accurate and do not aid in the least in the determination of the geometric capacity, since the shape of the curve near the origin can not be inferred from points so far removed. If there is to be an improvement in the determination of the geometric capacity by this method, it will come from measurements in the interval between 1000 and 100 cycles. For the better condensers this is hardly necessary, but would be of some advantage with the poorer ones.

To determine the geometric capacity by means of direct current measurements ${ }^{18}$ it is necessary to measure the apparent capacity at definite and very short times of discharge. With these values a curve is plotted in which the apparent capacities are ordinates and the times of discharge are abscissa. Extending this curve to cut the axis of zero time, the capacity thus indicated is the geometric capacity. Such curves in the case of three condensers are shown in Figs. I9 and 20. In each case four values were obtained with short times of discharge, the longest being a tenth of a second. There is also drawn for comparison the curve representing the alternating current capacities, where the period of the applied emf. is plotted as abscissa.

${ }^{18}$ This is similar to a method which was employed by Zeleny and Andrews, Phys. Rev. 27, p. 65; 1908. 


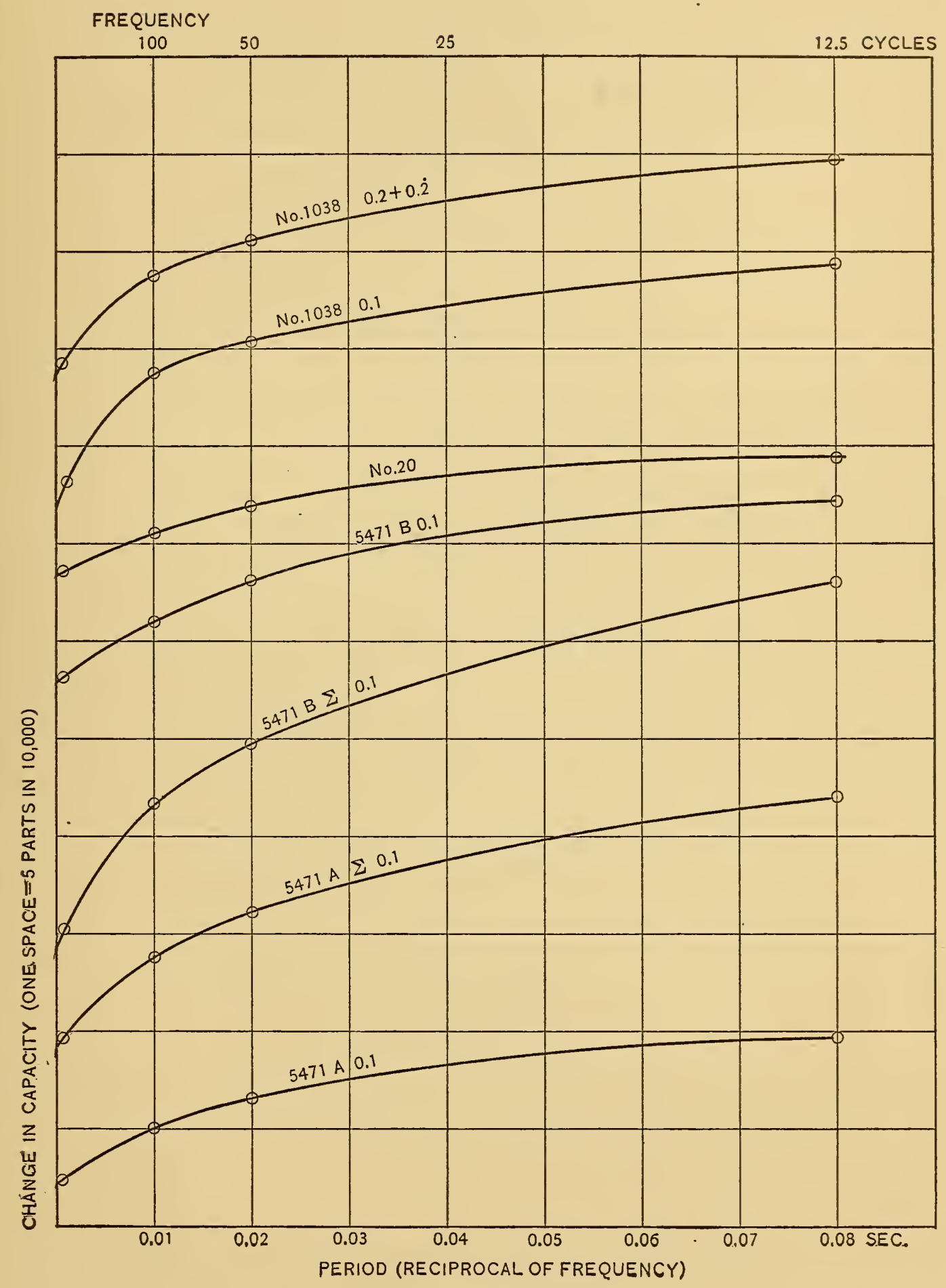

Fig. 18.-Curves showing the change of capacity at low frequency 
The extrapolation in the case of the D. C. capacities is much less satisfactory than with the $\mathrm{A}$. C. values, since the curve must be extended five times as far in the first case as in the second. However, it is sufficiently accurate to show that if there is any difference in the geometric capacity as determined by the two methods, it is very small; certainly less than a part in ten thousand, and in all probability less than a part in a hundred thousand.

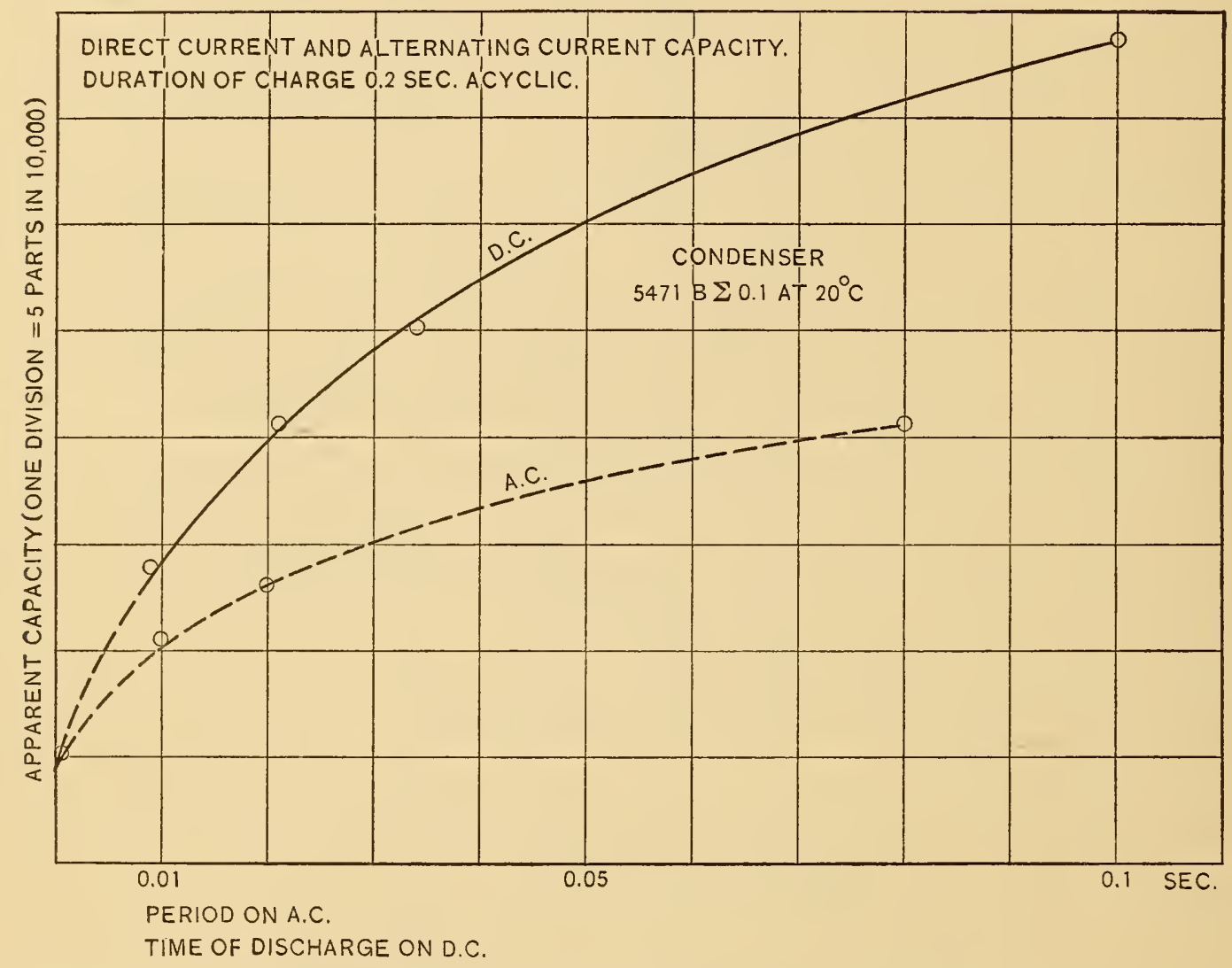

Fig. 19.-Curves showing agreement of the geometric capacity as determined by D. C. and A.C. measurements

\section{EFFECT OF LEAKAGE}

In the preceding work no account has been taken of the quantity of electricity conducted through the condenser. It is necessary to show that the resistance of the condensers is so high that this quantity is inappreciable. In the direct current work there was in each case an interval of a tenth of a second from the time the condensers were disconnected from the battery until they were discharged. In order that the leakage shall produce an effect of one part in a hundred thousand, a quantity equal to $E \times \mathrm{IO}^{-12}$ coulombs must leak through a tenth-microfarad condenser in o.I 
second. This requires that the resistance shall be $10^{11}$ ohms. As each of the condensers used, including the air condenser, had resistances higher than this, the effect of leakage was negligible in all the D. C. measurements. With A. C. the effect on the measured capacity is much less than with $\mathrm{D}$. C. It can even be shown that no appreciable part of the in-phase current in the case of the con-

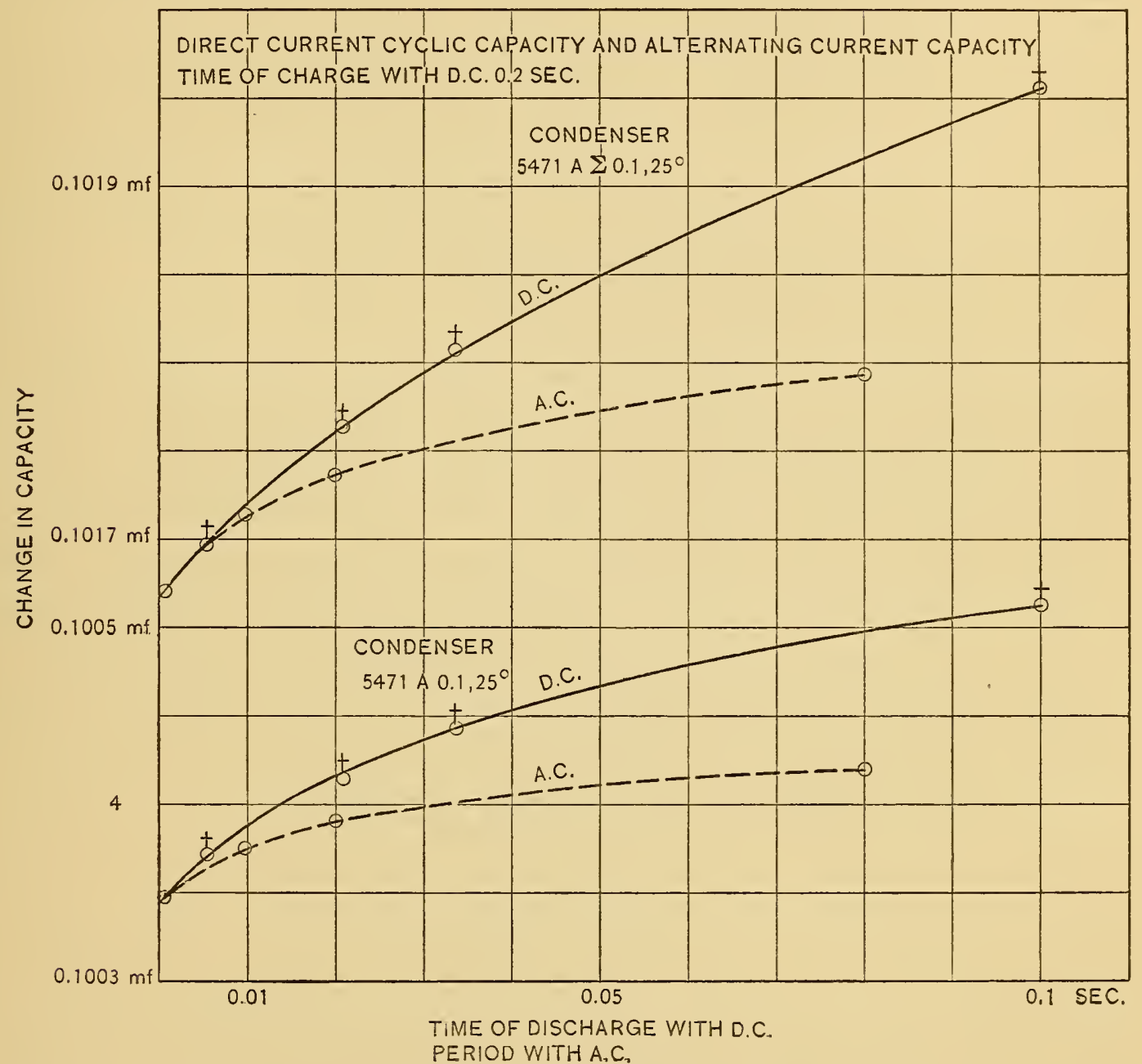

Fig. 20.-Curves showing the agreement of the geometric capacity as determined by $D . C$. and A. C. measurements

densers used in this investigation is due to leakage. Hence, the assumption that the insulation resistance is infinite is justifiable.

\section{POSSIBLE SOURCES OF ERROR IN THE MEASUREMENT OF THE GEOMETRIC CAPACITY}

Though the geometric capacity is the same whether determined by $\mathrm{D}$. C. or A. C., yet there remains the possibility that this agreement is only accidental. In this case there must be two or more 
causes whose effects exactly balance each other. There are three causes which may be operative to produce differences in the value of the geometric capacity by the two methods. The first lies in the possibility that, in the direct-current measurements, the total absorbed charge which is given up during the first instant of the discharge may be affected by the rate of dissipation of the free charge. The second is due to the fact that when alternating current of high frequency is used the distribution of the charge on the condenser plates is not uniform. The third lies in the possibility that the dielectric properties of mica are not the same with A. C. as with D. C., since with D. C. the charging or discharging may take place in a millionth of a second, while with $\mathrm{A}$. C. these processes take place much more slowly.

The effect of the rate of discharge of the free charge on the reappearance of the absorbed charge has been investigated experimentally. In the method of mixtures, if the resistance, $r$ (see Fig. 4), through which the condensers are discharged is varied over a wide range but always kept small enough so that no measurable part of the free charge will remain when the condensers are connected to the galvanometer, then if $C_{1}$ is an air condenser and $C_{2}$ a mica condenser, any change in the apparent capacity of $C_{2}$ on account of the variations of $r$ will be due to a change in the amount of the absorbed charge which is given up during the dissipation of the free charge. This will now be subjected to a numerical test. The quantity of the free charge $Q_{t}$ which is discharged in a time, $t$, is $Q_{t}=Q\left(\mathrm{I}-\mathrm{e}^{-\frac{t}{r c}}\right)$ where $Q$ is the total free charge. If $t$ is $\mathrm{O}$.I second, $\mathrm{c}=\mathrm{IO}-7$ farads, and $r=\mathrm{IO}^{5}$ ohms, $Q_{t}$ will differ from $Q$ by only four parts in a hundred thousand, so that for lower values of $r, Q_{t}$ is sensibly equal to $Q$. Experimentally it was found that when $r$ was changed from o.I ohm to rooo ohms no appreciable change in the apparent capacity occurred, but at $r=10000$ ohms there was a decrease of four parts in a hundred thousand when a medium-grade mica condenser was used. This shows that if $r$ is small this effect is negligible.

The effect of the frequency on the distribution of charge on the plates has been investigated mathematically by Coffin. ${ }^{19}$ An application of his formula to the air condensers used in these 


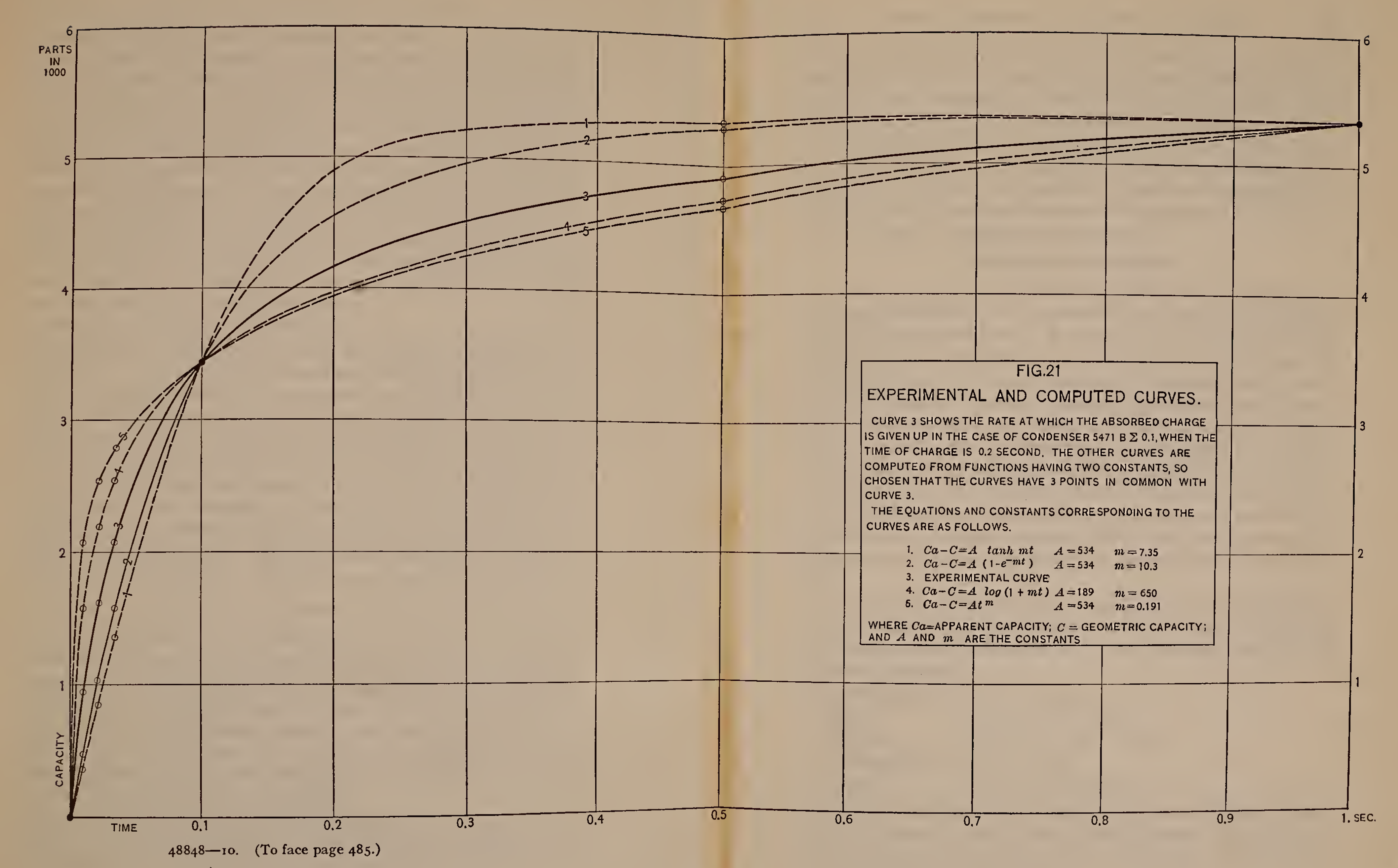


experiments showed that the change in capacity at 3000 cycles due to the fact that the charge is not uniformly distributed on the plates is of the magnitude of one part in a hundred million. With mica condensers it is of the same order of magnitude.

Since two of the three possible effects have been shown to produce negligible errors, the conclusion must be drawn that none of them enter. Hence, the geometric capacity as determined is a function of the dielectric constants and dimensions of the dielectrics of which the condenser is constructed.

\section{EMPIRICAL FORMULAS}

While a relationship between all of the various capacities has not been established, yet a method of procedure for an empirical treatment can be indicated. It is first necessary to determine the equation of the discharge curve for several different times of charge and then to find the relationship between these curves. In other words, the absorbed quantity of electricity which reappears in a given time must be expressed as a function of the time of charge and the time of discharge. Then the apparent capacity with different A. C. frequencies must be expressed as a function of the frequency and the relationship between this function and the previous one must be established. It will be seen that these requirements are rather rigorous; and, unless the functions are comparatively simple, a knowledge of them will be of no practical use.

The discharge curve with 0.2 second charge was determined for one condenser $(547 \mathrm{IB} \Sigma \mathrm{o} . \mathrm{r})$ with great care. An attempt was then made to find a formula with two constants which would represent this curve.

The following formulas were tried:

Pellat's ${ }^{20} \ldots \ldots \ldots C_{a}=C+A\left(\mathrm{I}-e^{-i m t}\right)$

Wilson's ${ }^{21} \ldots \ldots C_{a}=C+A \log (\mathrm{I}+m t)$

v. Schweidler's ${ }^{22} \ldots C_{a}=C+A t^{m}$

Curtis's........... $C_{a}=C+A \tanh m t$

In these equations $\mathrm{C}_{a}$ represents the apparent capacity, $C$ the geometric capacity, and $A$ and $m$ are constants. The curves to

${ }^{20}$ Ann. chim phys. (7), 18, p. I50; 1899.

${ }^{21}$ Proc. Roy. Soc. A., 82, p. 409; 1909.

${ }^{22}$ Ann. d. Phys., 24, p. 7 Ir; 1907. 
represent these equations, together with the experimental curve, are given in Fig. 2I. The constants of each equation have been so chosen that the curve which it represents passes through the same points as the experimental curve when $t=0$.I second and when $t=\mathrm{I}$ second. It is seen that, for small values of $t$, the difference between the ordinates of the experimental and empirical curves is much larger than the experimental error.

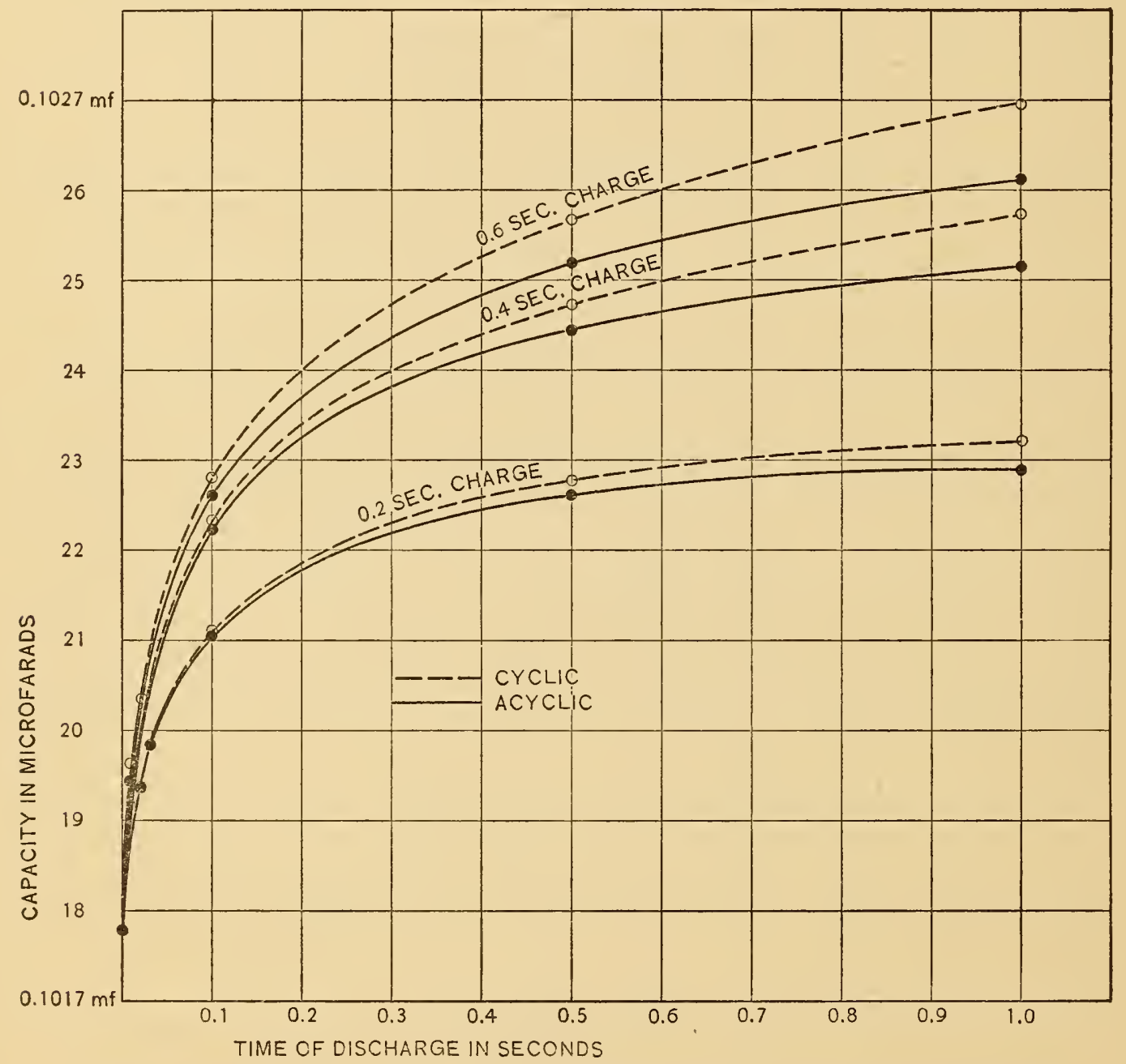

Fig. 22.-Cyclic and acyclic capacity with different times of charge and of discharge

By taking a series of terms any one of the above functions can be extended so as to represent the curve with any desired accuracy. However, such a function is so complicated that it does not seem feasible to attempt to express all the different D. C. and A. C. values of the capacity by means of a single function. ${ }^{23}$

${ }^{23} \mathrm{Hill}$ has found the same difficulty in the case of paper condensers, Phys. Rev., 26, p. $400 ; 1908$. 


\section{CASES OF PRACTICAL IMPORTANCE}

There is one case of considerable practical importance where it is possible to pass from direct current to alternating current values with a fair degree of accuracy. If a good grade mica condenser is measured by means of a rotating commutator or other means for charging and discharging a given number of times per second, the capacity so determined will not differ greatly from the value determined by alternating current of the same frequency. It is evident that this agreement will be closer the higher the frequency.

A considerable number of comparisons at Ioo cycles have been made, and the measured differences seldom exceed two or three parts in ten thousand. They may be either positive or negative, depending upon the length of contact.

One may be reasonably sure that with good mica condensers the difference of the A. C. and D. C. values of the capacity at any one frequency will be less than a tenth of I per cent.

Another case of some interest is the difference between the cyclic and acyclic values of the capacity at different times of charge and different times of discharge. This is illustrated in the curves of Fig. 22. It is evident that this difference is also a function of the length of the cycle, though this is not shown in the curves.

\section{SUMIMARY.}

(I) The various methods for measuring capacity with A. C. and D. C. are discussed, and the best methods to use when mica condensers are to be measured are indicated.

(2) A method is given by which the temperature coefficient of a mica condenser may be made very small, at most a few parts in a hundred thousand per degree.

(3) The effect of changes of the atmospheric pressure upon the capacity are shown to be small, though in some cases they are not negligible.

(4) An explanation of the change of capacity with voltage, which is observed in the case of silvered-mica condensers, is offered.

(5) Condensers which are kept in a vacuum and maintained at constant temperature can be relied upon to a few parts in a hundred thousand. Such condensers are somewhat more stable than condensers which are maintained at constant temperature, 
but subjected to the ordinary fluctuations of atmospheric pressure. Condensers exposed to the ordinary changes of room temperature show unaccountable variations of the capacity of two or three parts in ten thousand.

(6) It is shown that, taking the measured values of the specific resistances of mica and paraffin, Maxwell's theory of a stratified dielectric does not account for the absorption actually observed in the case of a mica condenser. This theory is then applied to alternating current measurements, and it is shown that, according to this theory, the loss of energy per second in the dielectric is nearly independent of the frequency, whereas measurements show that the loss of energy per cycle is almost independent of the frequency. The conclusion is drawn that this theory alone will not explain the phenomena of absorption.

(7) The capacity of a condenser with A. C. of infinite frequency is shown to be the same as the capacity with $\mathrm{D}$. C. when the time of discharge is infinitely short. This capacity is called the geometric capacity, since it is a function of the dimensions and dielectric constants of the dielectrics.

In conclusion, I wish to express my thanks to Dr. E. B. Rosa and to Dr. F. W. Grover for the interest they have shown in this research, and for the many valuable suggestions they have given.

Washington, D. C., April 22, i9io. 